\title{
Research Article \\ On Some Types of Multigranulation Covering Based on Binary Relations
}

\author{
Ashraf Nawar ${ }^{1}{ }^{1}$ and E. A. Elsakhawy ${ }^{2}$ \\ ${ }^{1}$ Department of Mathematics and Computer Science, Faculty of Science, Menoufia University, Menoufia, Egypt \\ ${ }^{2}$ Department of Mathematics, Faculty of Science, Ain Shams University, Abbassia, Cairo, Egypt \\ Correspondence should be addressed to Ashraf Nawar; ashraf_nawar2020@yahoo.com
}

Received 9 June 2021; Revised 16 July 2021; Accepted 2 August 2021; Published 8 November 2021

Academic Editor: Serena Matucci

Copyright (c) 2021 Ashraf Nawar and E. A. Elsakhawy. This is an open access article distributed under the Creative Commons Attribution License, which permits unrestricted use, distribution, and reproduction in any medium, provided the original work is properly cited.

\begin{abstract}
Recently, the notions of right and left covering rough sets were constructed by right and left neighborhoods to propose four types of multigranulation covering rough set (MGCRS) models. These models were constructed using the granulations as equivalence relations. In this paper, we introduce four types of multigranulation covering rough set models under arbitrary relations using the $q$-minimal and $q$-maximal descriptors of objects in a given universe. We also study the properties of these new models. Thus, we explore the relationships between these models. Then, we put forward an algorithm to illustrate the method of reduction based on the presented model. Finally, we give an illustrative example to show its efficiency and importance.
\end{abstract}

\section{Introduction}

The notion of rough set theory originated by Pawlak in 1982 $[1,2]$ to deal with uncertain information and knowledge. It is a tool concerned with the approximation of sets described by a single binary relation. In the view of granular computing suggested by Zadeh [3], a general concept described by a set is characterized via the upper and lower approximations under a single granulation (always equivalence relation) on the universe. This tool has been widely used in many subjects including machine learning, data mining, decision support, and analysis. In the past 20 years, many authors have proposed several extensions of the rough set model [4-19]. In some cases, it is important to use multiequivalence relations on the universe to describe precisely a target concept. Recently, more attention is given to multigranulation rough set (MGRS) models and, also, to multigranulation covering rough set (MGCRS) models in which a target concept is approximated by employing the maximal or minimal descriptors of objects in the given universe. In [20, 21], Qian et al. developed a multigranulation rough set (MGRS) model by using equivalence relations. Several scholars worked on MGRS such as the MGRS model through multiple tolerance relations in incomplete information systems, MGRS via the fuzzy approximation space, the hierarchical structures of MGRS, the topological and lattice-theoretic properties of MGRS, and the efficient rough feature selection algorithm with MGRS [22-30]. Moreover, Liu and Miao and Liu and Wang $[31,32]$ introduced the multigranulation covering via rough set (MGCRS) and fuzzy rough set (MGCFRS). Lin et al. studied two types of the neighborhood via MGRS [33] and three new types of MGCRS [34]. Also, three types of MGRS via the tolerance, ordered, and generalized relations are investigated and developed the multigranulation decision-theoretic rough set [35-38]. In addition, Liu et al. [39] proposed four new types of MGCRS using the minimal and maximal descriptions and discussed relevant characteristics. For more details about MGRS, see, for instance, [40-44].

The notions of left and right covering rough sets proposed by Abd El-Monsef et al. [45] are important tool to make an extension of Liu et al. [39]. The objective of this paper is to develop new models of MGCRS using the notions of left and right covering using the concepts of $q$-minimal and $q$-maximal descriptions. Also, we discuss the properties of these models. The relationships between these models are studied. Then, we present the reduction method over 
our proposed work and establish a numerical example to show its performance. The paper consists of six sections and is organized as follows: Section 1 deals with a brief history to the subject. Section 2 includes the preliminary concepts. Section 3 is the main core of the paper and consists of the new models. In Section 4, the properties and differences between the proposed models are introduced. Section 5 explores new criteria to make a reduction with a test example. We end up with conclusion in the last section.

\section{Basic Terminologies and Results}

This section provides a short survey of some notions used throughout the article.

Definition 1 [26]. Let $\mathbb{Q}$ be an universal set and $\varnothing \neq \mathbb{E}=\left\{\tilde{\mathbb{E}}_{1}\right.$, $\left.\tilde{\mathbb{E}}_{2}, \cdots, \tilde{\mathbb{E}}_{m}\right\} \subseteq \mathbb{Q}$. We call $\mathbb{E}$ as a covering of $\mathbb{Q}$, if $\bigcup_{i=1}^{m} \tilde{\mathbb{E}}_{i}(w)$ $=\mathbb{Q}$ for any $w \in \mathbb{Q}$. Also, $(\mathbb{Q}, \mathbb{E})$ is called a covering approximation space (briefly, CAS).

Definition 2 [46]. Let $\mathscr{R}$ be a binary relation on an universe set $\mathbb{Q}$, and for every $w \in \mathbb{Q}$, we have the following two classes. Define the after and fore sets as follows:

$$
\begin{aligned}
& w \mathscr{R}=\{v \in \mathbb{Q}: w \mathscr{R} v\}, \\
& \mathscr{R} w=\{v \in \mathbb{Q}: v \mathscr{R} w\} .
\end{aligned}
$$

Definition 3 [45]. Let $\mathscr{R}$ be a binary relation on an universe set $\mathbb{Q}$. For each $w \in \mathbb{Q}$, define the right covering $\mathscr{C}_{\mathbf{r}}$ (resp., the left covering $\mathscr{C}_{1}$ ) as follows:

$$
\begin{aligned}
& \mathscr{C}_{\mathbf{r}}=\left\{w \mathscr{R}: \mathbb{Q}=\bigcup_{w \in \mathbb{Q}} w \mathscr{R}\right\}, \\
& \mathscr{C}_{\mathbf{1}}=\left\{\mathscr{R} w: \mathbb{Q}=\bigcup_{w \in \mathbb{Q}} \mathscr{R} w\right\} .
\end{aligned}
$$

Definition 4 [45]. Let $\mathscr{R}$ be a binary relation on an universe set $\mathbb{Q}$ and $\mathbb{E}_{q}$ be a $q$-cover of $\mathbb{Q}$, where $q \in\{\mathbf{r}, \mathbf{l}\}$. Then, $(\mathbb{Q}$, $\mathscr{R}, \mathbb{E}_{q}$ ) is said to be $\mathbb{E}_{q}$ covering approximation space (briefly, $\mathbb{E}_{q}$-CAS).

Definition 5 [45]. Let $\left(\mathbb{Q}, \mathscr{R}, \mathbb{E}_{q}\right)$ be $\mathbb{E}_{q}$-CAS. For every $w \in \mathbb{Q}$ , define the right neighborhood $\mathbb{N}_{r}$, the left neighborhood $\mathbb{N}_{l}$, the intersection neighborhood $\mathbb{N}_{\mathrm{i}}$, and the union neighborhood $\mathbb{N}_{\mathrm{u}}$, respectively, as follows:

$$
\begin{aligned}
& \mathbb{N}_{\mathrm{r}}(w)=\left\{\mathbf{C} \in \mathscr{C}_{\mathrm{r}}: w \in \mathbf{C}\right\} \\
& \mathbb{N}_{\mathrm{l}}(w)=\left\{\mathbf{C} \in \mathscr{C}_{\mathrm{l}}: w \in \mathbf{C}\right\} \\
& \mathbb{N}_{\mathrm{i}}(w)=\mathbb{N}_{\mathrm{r}}(w) \cap \mathbb{N}_{\mathrm{l}}(w), \\
& \mathbb{N}_{\mathrm{u}}(w)=\mathbb{N}_{\mathrm{r}}(w) \cup \mathbb{N}_{\mathrm{l}}(w)
\end{aligned}
$$

Definition 6 [45]. Let $\left(\mathbb{Q}, \mathscr{R}, \mathbb{E}_{q}\right)$ be $\mathbb{E}_{q}$-CAS and $\forall p \in\{\mathbf{r}, \mathbf{l}, \mathbf{i}$ , u $\}$ and $\mathscr{Z} \subseteq \mathbb{Q}$. Define the $p$-lower approximation, $p$-upper approximation, $p$-boundary, $p$-positive, $p$-negative, and $p$ accuracy of $\mathscr{Z}$, respectively, as follows:

$$
\begin{aligned}
\mathbf{L}_{p}(\mathscr{Z}) & =\left\{w \in \mathscr{Z}: \mathbb{N}_{p}(w) \subseteq \mathscr{Z}\right\}, \\
\mathbf{U}_{p}(\mathscr{Z}) & =\left\{w \in \mathscr{Z}: \mathbb{N}_{p}(w) \cap \mathscr{Z} \neq \varnothing\right\}, \\
B_{p}(\mathscr{Z}) & =\mathbf{U}_{p}(\mathscr{Z})-\mathbf{L}_{p}(\mathscr{Z}), \\
\oplus_{p}(\mathscr{Z}) & =\mathbf{L}_{p}(\mathscr{Z}), \\
\ominus_{p}(\mathscr{Z}) & =\mathbb{Q}-\mathbf{U}_{p}(\mathscr{Z}) \\
A_{p}(\mathscr{Z}) & =\frac{\left|\mathbf{L}_{p}(\mathscr{Z})\right|}{\left|\mathbf{U}_{p}(\mathscr{Z})\right|}, \quad \text { where }\left|\mathbf{U}_{p}(\mathscr{Z})\right| \neq 0 .
\end{aligned}
$$

Pawlak's $[1,2]$ rough set properties are given as follows: $\left(L_{1}\right) \mathbf{L}(\mathscr{Z}) \subseteq \mathscr{Z},\left(H_{1}\right) \mathscr{Z} \subseteq \mathbf{U}(\mathscr{Z})$.

$\left(L_{2}\right) \mathbf{L}(\mathbb{Q})=\mathbb{Q},\left(H_{2}\right) \mathbf{U}(\varnothing)=\varnothing$.

$\left(L_{3}\right) \mathbf{L}(\varnothing)=\varnothing,\left(H_{3}\right) \mathbf{U}(\mathbb{Q})=\mathbb{Q}$.

$\left(L_{4}\right)$ If $\mathscr{Z}_{1} \subseteq \mathscr{Z}_{2}$, then $\mathbf{L}\left(\mathscr{Z}_{1}\right) \subseteq \mathbf{L}\left(\mathscr{Z}_{2}\right),\left(H_{4}\right) \mathbf{U}\left(\mathscr{Z}_{1}\right) \subseteq \mathbf{U}\left(\mathscr{Z}_{2}\right)$.

$\left(L_{5}\right) \quad \mathbf{L}\left(\mathscr{Z}_{1} \cap \mathscr{Z}_{2}\right)=\mathbf{L}\left(\mathscr{Z}_{1}\right) \cap \mathbf{L}\left(\mathscr{Z}_{2}\right) \cdot\left(H_{5}\right) \mathbf{U}\left(\mathscr{Z}_{1} \cup \mathscr{Z}_{2}\right)=$ $\mathbf{U}\left(\mathscr{Z}_{1}\right) \cup \mathbf{U}\left(\mathscr{Z}_{2}\right)$.

$\left(L_{6}\right) \quad \mathbf{L}\left(\mathscr{Z}_{1} \cup \mathscr{Z}_{2}\right) \supseteq \mathbf{L}\left(\mathscr{Z}_{1}\right) \cup \mathbf{L}\left(\mathscr{E}_{2}\right) \cdot\left(H_{6}\right) \mathbf{U}\left(\mathscr{Z}_{1} \cap \mathscr{Z}_{2}\right) \subseteq$ $\mathbf{U}\left(\mathscr{Z}_{1}\right) \cap \mathbf{U}\left(\mathscr{Z}_{2}\right)$.

$\left(L_{7}\right) \mathbf{L}\left(\mathscr{Z}^{c}\right)=(\mathbf{U}(\mathscr{Z}))^{c},\left(H_{7}\right) \mathbf{U}\left(\mathscr{Z}^{c}\right)=(\mathbf{L}(\mathscr{Z}))^{c}$.

$\left(L_{8}\right) \mathbf{L}(\mathbf{L}(\mathscr{Z}))=\mathbf{L}(\mathscr{Z}),\left(H_{8}\right) \mathbf{U}(\mathbf{U}(\mathscr{Z}))=\mathbf{U}(\mathscr{Z})$.

$\left(L_{9}\right) \mathbf{L}(\mathbf{L}(\mathscr{Z}))^{c}=(\mathbf{L}(\mathscr{Z}))^{c},\left(L_{9}\right) \mathbf{U}(\mathbf{U}(\mathscr{Z}))^{c}=(\mathbf{U}(\mathscr{Z}))^{c}$.

Definition 7 [47]. Let $(\mathbb{Q}, \mathbb{E})$ be a CAS and $\mathscr{Z} \subseteq \mathbb{Q}$. For any $w \in \mathbb{Q}$, define the minimal and maximal descriptions of $w$, respectively, as follows:

$$
\begin{aligned}
& \mathcal{H}_{\mathbb{E}}=\{\mathcal{C} \in \mathbb{E}: w \in \mathcal{C} \wedge(\forall \mathcal{S} \in \mathbb{E} \wedge w \in \mathcal{S} \wedge \mathcal{S} \subseteq \mathcal{C} \Rightarrow \mathcal{S}=\mathcal{C})\}, \\
& \mathscr{D}_{\mathbb{E}}=\{\mathscr{C} \in \mathbb{E}: w \in \mathscr{C} \wedge(\forall \mathcal{S} \in \mathbb{E} \wedge w \in \mathcal{S} \wedge \mathcal{S} \supseteq \mathscr{C} \Rightarrow \mathcal{S}=\mathscr{C})\} .
\end{aligned}
$$

Definition 8 [39]. Let $(\mathbb{Q}, \mathbb{E})$ be MGCAS and $\mathscr{Z} \subseteq \mathbb{Q}$. For any $w \in \mathbb{Q}$, define four types of the lower and upper approximations, respectively, as follows:

$$
\begin{aligned}
& \sum_{d=1}^{\mathbf{L}^{1}{ }_{n}}(\mathcal{Z})=\left\{w \in \mathbb{Q}: \cap \mathcal{H}_{\mathbb{E}_{1}}(w) \subseteq \mathcal{Z} \text { or } \cap \mathcal{H}_{\mathbb{E}_{2}}(w) \subseteq \mathcal{Z}\right. \\
& \text { or } \left.\cdots \text { or } \cap \mathcal{H}_{\mathbb{E}_{n}}(w) \subseteq \mathcal{Z}\right\} \text {, } \\
& \sum_{d=1}^{\mathbf{U}^{1}} \mathbb{E}_{d}(\mathcal{Z})=\left\{w \in \mathbb{Q}:\left[\cap \mathcal{H}_{\mathbb{E}_{1}}(w)\right] \cap \mathcal{Z} \neq \varnothing\right. \\
& \text { and }\left[\cap \mathcal{H}_{\mathbb{E}_{2}}(w)\right] \cap \mathcal{Z} \neq \varnothing \\
& \text { and } \left.\cdots \text { and }\left[\cap \mathcal{H}_{\mathbb{E}_{n}}(w)\right] \cap \mathcal{Z} \neq \varnothing\right\} \text {, } \\
& \mathbf{L}_{\mathbb{E}_{d}}^{2}(\mathcal{Z})=\left\{w \in \mathbb{Q}: \cup \mathcal{H}_{\mathbb{E}_{1}}(w) \subseteq \mathcal{Z} \text { or } \cup \mathcal{H}_{\mathbb{E}_{2}}(w) \subseteq \mathcal{Z}\right. \\
& \text { or } \left.\cdots \text { or } \cup \mathcal{H}_{\mathbb{E}_{n}}(w) \subseteq \mathcal{Z}\right\} \text {, }
\end{aligned}
$$




$$
\begin{aligned}
& \mathbf{U}_{\mathbb{E}_{d}}^{2}(\mathcal{Z})=\left\{w \in \mathbb{Q}:\left[\cup_{\mathcal{H}_{\mathbb{E}_{1}}}(w)\right] \cap \mathcal{Z} \neq \varnothing\right. \\
& \text { and }\left[\cup \mathcal{H}_{\mathbb{E}_{2}}(w)\right] \cap \mathcal{Z} \neq \varnothing \\
& \text { and } \left.\cdots \text { and }\left[\cup \mathcal{H}_{\mathbb{E}_{n}}(w)\right] \cap \mathcal{Z} \neq \varnothing\right\} \text {, } \\
& \mathbf{L}_{\mathbb{E}_{d}}^{3}(\mathscr{Z})=\left\{w \in \mathbb{Q}: \cap \mathcal{D}_{\mathbb{E}_{1}}(w) \subseteq \mathscr{Z} \text { or } \cap \mathcal{D}_{\mathbb{E}_{2}}(w) \subseteq \mathscr{Z}\right. \\
& \text { or } \left.\cdots \text { or } \cap \mathcal{D}_{\mathbb{E}_{n}}(w) \subseteq \mathscr{Z}\right\} \text {, } \\
& \mathbf{U}_{\mathbb{E}_{d}}^{3}(\mathscr{Z})=\left\{w \in \mathbb{Q}:\left[\cap \mathcal{D}_{\mathbb{E}_{1}}(w)\right] \cap \mathscr{Z} \neq \varnothing\right. \\
& \text { and }\left[\cap \mathcal{D}_{\mathbb{E}_{2}}(w)\right] \cap \mathscr{Z} \neq \varnothing \\
& \text { and } \left.\cdots \text { and }\left[\cap \mathcal{D}_{\mathbb{E}_{n}}(w)\right] \cap \mathscr{Z} \neq \varnothing\right\} \text {, } \\
& \mathbf{L}_{\mathbb{E}_{d}}^{4}(\mathscr{Z})=\left\{w \in \mathbb{Q}: \cup \mathcal{D}_{\mathbb{E}_{1}}(w) \subseteq \mathscr{Z} \text { or } \cup \mathcal{D}_{\mathbb{E}_{2}}(w) \subseteq \mathscr{Z}\right. \\
& \text { or } \left.\cdots \text { or } \cup \mathcal{D}_{\mathbb{E}_{n}}(w) \subseteq \mathscr{Z}\right\} \text {, } \\
& \mathbf{U}_{\mathbb{E}_{d}}^{4}(\mathscr{Z})=\left\{w \in \mathbb{Q}:\left[\cup \mathcal{D}_{\mathbb{E}_{1}}(w)\right] \cap \mathscr{Z} \neq \varnothing\right. \\
& \text { and }\left[\cup \mathcal{D}_{\mathbb{E}_{2}}(w)\right] \cap \mathscr{Z} \neq \varnothing \\
& \text { and } \left.\cdots \text { and }\left[\cup \mathcal{D}_{\mathbb{E}_{n}}(w)\right] \cap \mathscr{Z} \neq \varnothing\right\} \text {, }
\end{aligned}
$$

If $\mathbf{L}_{\mathbb{E}_{d}}^{1}(\mathscr{Z})$ (resp., $\mathbf{L}_{\mathbb{E}_{d}}^{2}(\mathscr{Z}), \mathbf{L}_{\mathbb{E}_{d}}^{3}(\mathscr{Z})$, and $\mathbf{L}_{\mathbb{E}_{d}}^{4}(\mathscr{Z})$ ) $\neq$ $\mathbf{U}_{\mathbb{E}_{d}}^{1}(\mathscr{Z})$ (resp., $\mathbf{U}_{\mathbb{E}_{d}}^{2}(\mathscr{Z}), \mathbf{U}_{\mathbb{E}_{d}}^{3}(\mathscr{Z})$, and $\mathbf{U}_{\mathbb{E}_{d}}^{4}(\mathscr{Z})$ ), then $\mathscr{Z}$ is called the first kind of a multigranulation covering rough set (briefly, type 1-MGCRS) (resp., type 2-MGCRS, type 3MGCRS, and type 4-MGCRS), else it is definable.

Definition 9 [48]. Let $(\mathbb{Q}, \mathbb{E})$ be a covering information system. For any $\mathscr{Z} \subseteq \mathbb{Q}$ and $w \in \mathbb{Q}$, define the first type of optimistic multigranulation covering lower approximation (briefly, 1-OMGCLA) ${ }_{1} \mathbf{L}_{\sum_{d=1}^{n}{ }_{d} \mathbb{E}}^{O}(\mathcal{Z})$ and the first type of optimistic multigranulation covering upper approximation (briefly, 1-OMGCUA) ${ }_{1} \mathbf{U}_{\sum_{d=1}^{n} d}^{O} \mathbb{E}(\mathcal{Z})$ as follows:

$$
\begin{gathered}
\sum_{d=1} \mathbf{L}_{d^{n}}^{O}(\mathscr{E})=\left\{w \in \mathbb{Q}:(w)_{\mathbb{E}}^{1} \subseteq \mathscr{Z} \vee(w)_{\mathbb{E}}^{2} \subseteq \mathscr{Z} \vee \cdots \vee(w)_{\mathbb{E}}^{n} \subseteq \mathscr{Z}\right\}, \\
{ }_{1} \mathbf{L}_{{ }_{d=1}^{O}{ }_{d}{ }_{d} \mathbb{E}}(\mathscr{E})=\left\{w \in \mathbb{Q}:(w)_{\mathbb{E}}^{1} \cap \mathscr{Z} \neq \varnothing \wedge(w)_{\mathbb{E}}^{2}\right. \\
\left.\cap \mathscr{E} \neq \varnothing \wedge \cdots \wedge(w)_{\mathbb{E}}^{n} \cap \mathscr{Z} \neq \varnothing\right\} .
\end{gathered}
$$

Definition 10 [48]. Let $(\mathbb{Q}, \mathbb{E})$ be a covering information system. For any $\mathscr{Z} \subseteq \mathbb{Q}$ and $w \in \mathbb{Q}$, define the first type of pessimistic multigranulation covering lower approximation (briefly, 1-PMGCLA) ${ }_{1} \mathbf{L}_{\sum_{d=1}^{n} d_{\mathbb{E}} \mathbb{E}_{q}}^{P}(\mathscr{Z})$ and the first type of pessimistic multigranulation covering upper approximation

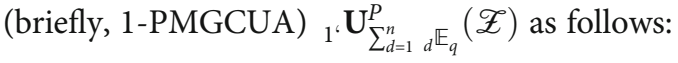

$$
\begin{aligned}
\sum_{d=1} \mathbf{L}^{P}{ }_{n}(\mathscr{E})= & \left\{w \in \mathbb{Q}:(w)_{\mathbb{E}}^{1} \subseteq \mathscr{Z} \wedge(w)_{\mathbb{E}}^{2} \subseteq \mathscr{E} \wedge \cdots \wedge(w)_{\mathbb{E}}^{n} \subseteq \mathscr{Z}\right\}, \\
{ }_{1} \mathbf{U}^{P}{ }_{{ }^{n}{ }_{d=1}{ }_{d} \mathbb{E}}(\mathscr{Z})= & \left\{w \in \mathbb{Q}:(w)_{\mathbb{E}}^{1} \cap \mathscr{Z} \neq \varnothing \vee(w)_{\mathbb{E}}^{2}\right. \\
& \left.\cap \mathscr{Z} \neq \varnothing \vee \cdots \vee(w)_{\mathbb{E}}^{n} \cap \mathscr{Z} \neq \varnothing\right\} .
\end{aligned}
$$

Next, we have the following definitions using the notion of $\mathbb{E}_{q}$-CAS.

Definition 11. Let $\left(\mathbb{Q}, \mathscr{R}, \mathbb{E}_{q}\right)$ be $\mathbb{E}_{q}$-CAS and $\mathscr{Z} \subseteq \mathbb{Q}$. For any $w \in \mathbb{Q}$, define the $q$-minimal and $q$-maximal descriptions of $w$, respectively, as follows:

$$
\begin{aligned}
& \mathcal{H}_{\mathbb{E}_{q}}=\left\{\mathcal{C} \in \mathbb{E}_{q}: w \in \mathcal{C} \wedge\left(\forall \mathcal{S} \in \mathbb{E}_{q} \wedge w \in \mathcal{S} \wedge \mathcal{S} \subseteq \mathcal{C} \Rightarrow \mathcal{S}=\mathcal{C}\right)\right\}, \\
& \mathscr{D}_{\mathbb{E}_{q}}=\left\{\mathscr{C} \in \mathbb{E}_{q}: w \in \mathscr{C} \wedge\left(\forall \mathcal{S} \in \mathbb{E}_{q} \wedge w \in \mathcal{S} \wedge \mathcal{S} \supseteq \mathscr{C} \Rightarrow \mathcal{S}=\mathscr{C}\right)\right\} .
\end{aligned}
$$

We give the following example to illustrate the above definition.

Example 1. Let $\left(\mathbb{Q}, \mathscr{R}, \mathbb{E}_{q}\right)$ be $\mathbb{E}_{q}$-CAS, $\mathbb{Q}=\left\{k_{1}, k_{2}, k_{3}, k_{4}\right\}$ and $\mathscr{R}=\left\{\left(k_{1}, k_{4}\right),\left(k_{2}, k_{2}\right),\left(k_{2}, k_{3}\right),\left(k_{3}, k_{2}\right),\left(k_{4}, k_{1}\right),\left(k_{4}, k_{3}\right)\right\}$. Then, we have the following results:

$$
\begin{aligned}
& \mathcal{H}_{\mathbb{E}_{\mathrm{r}}}\left(k_{1}\right)=\left\{k_{1}, k_{3}\right\}, \mathcal{H}_{\mathbb{E}_{\mathrm{r}}}\left(k_{2}\right)=\left\{k_{2}\right\}, \\
& \mathcal{H}_{\mathbb{E}_{\mathrm{r}}}\left(k_{3}\right)=\left\{\left\{k_{1}, k_{3}\right\},\left\{k_{2}, k_{3}\right\}\right\}, \mathcal{H}_{\mathbb{E}_{\mathrm{r}}}\left(k_{4}\right)=\left\{k_{4}\right\}, \\
& \mathcal{H}_{\mathbb{E}_{1}}\left(k_{1}\right)=\left\{k_{1}\right\}, \mathcal{H}_{\mathbb{E}_{1}}\left(k_{2}\right)=\left\{\left\{k_{2}, k_{3}\right\},\left\{k_{2}, k_{4}\right\}\right\}, \\
& \mathcal{H}_{\mathbb{E}_{1}}\left(k_{3}\right)=\left\{\left\{k_{2}, k_{3}\right\}\right\}, \mathcal{H}_{\mathbb{E}_{1}}\left(k_{4}\right)=\left\{k_{4}\right\}, \\
& \mathscr{D}_{\mathbb{E}_{\mathrm{r}}}\left(k_{1}\right)=\left\{k_{1}, k_{3}\right\}, \mathscr{D}_{\mathbb{E}_{\mathrm{r}}}\left(k_{2}\right)=\left\{k_{2}, k_{3}\right\}, \\
& \mathscr{D}_{\mathbb{E}_{\mathrm{r}}}\left(k_{3}\right)=\left\{\left\{k_{1}, k_{3}\right\}\left\{k_{2}, k_{3}\right\}\right\}, \mathscr{D}_{\mathbb{E}_{\mathrm{r}}}\left(k_{4}\right)=\left\{k_{4}\right\}, \\
& \mathscr{D}_{\mathbb{E}_{1}}\left(k_{1}\right)=\left\{k_{1}\right\}, \mathscr{D}_{\mathbb{E}_{1}}\left(k_{2}\right)=\left\{\left\{k_{2}, k_{3}\right\},\left\{k_{2}, k_{4}\right\}\right\}, \\
& \mathscr{D}_{\mathbb{E}_{1}}\left(k_{3}\right)=\left\{k_{2}, k_{3}\right\}, \mathscr{D}_{\mathbb{E}_{1}}\left(k_{4}\right)=\left\{k_{2}, k_{4}\right\} .
\end{aligned}
$$

Definition 12 . Let $\left(\mathbb{Q}, \mathscr{R}, \mathbb{E}_{q}\right)$ be $\mathbb{E}_{q}$-CAS and $\mathscr{Z} \subseteq \mathbb{Q}$. For any $w \in \mathbb{Q}$, define the lower and upper approximations, respectively, as follows:

$$
\begin{aligned}
\mathbf{L}_{\mathbb{E}_{q}}(\mathcal{Z}) & =\left\{w \in \mathcal{Z}:\left(\cap \mathcal{H}_{\mathbb{E}_{q}}(w)\right) \subseteq \mathcal{Z}\right\}, \\
\mathbf{U}_{\mathbb{E}_{q}}(\mathscr{Z}) & =\left\{w \in \mathbb{Q}:\left(\cap \mathscr{D}_{\mathbb{E}_{q}}(w)\right) \cap \mathscr{Z} \neq \varnothing\right\} .
\end{aligned}
$$

To explain the above definition, we give the following example.

Example 2. Consider Example 1, if $\mathscr{Z}=\left\{k_{1}, k_{2}, k_{4}\right\}$, then we have the following results.

$$
\begin{aligned}
& \mathbf{L}_{\mathbb{E}_{\mathrm{r}}}(\mathscr{Z})=\left\{k_{2}, k_{4}\right\}, \\
& \mathbf{U}_{\mathbb{E}_{\mathrm{r}}}(\mathscr{Z})=\left\{k_{1}, k_{2}, k_{4}\right\}, \\
& \mathbf{L}_{\mathbb{E}_{1}}(\mathscr{Z})=\left\{k_{1}, k_{2}, k_{4}\right\}, \\
& \mathbf{U}_{\mathbb{E}_{1}}(\mathscr{Z})=\mathbb{Q} .
\end{aligned}
$$




\section{Multi- $\mathbb{E}_{q}-$ Covering Approximation Space}

Presume that $\mathbb{Q}$ is an universal set, $\Re$ is a family of binary relations on $\mathbb{Q}$, and $\mathbb{E}_{q}$ is $q$-cover of $\mathbb{Q}$ depending on $\mathfrak{R}$, where $q \in\{\mathbf{l}, \mathbf{r}\}$. Thus, $\left(\mathbb{Q}, \mathfrak{R}, E_{q}\right)$ is called a multi- $\mathbb{E}_{q}$-covering approximation space (briefly, $M \mathbb{E}_{q} \mathrm{CAS}$ ).

Definition 13. Assume that $\left(\mathbb{Q}, \mathfrak{R}, \mathbb{E}_{q}\right)$ is a $\mathrm{ME}_{q} \mathrm{CAS}$ and $\mathfrak{R}=$ $\left\{\mathscr{R}_{1}, \mathscr{R}_{2}, \cdots, \mathscr{R}_{S}\right\}, \forall S \in I$, for any $\mathscr{Z} \subseteq \mathbb{Q}$ and $w \in \mathbb{Q}$. Then, we have four novel kinds of lower and upper approximations written as follows:

Style 1

The 1-MCLA ${ }_{1} \mathbf{L}_{\sum_{d=1}^{n} d \mathbb{E}_{q}}(\mathscr{E})$ and the 1-MCUA ${ }_{1} \mathbf{L}_{\sum_{d=1 d}^{n} \mathbb{E}_{q}}$ $(\mathscr{Z})$ are shown as follows:

$$
\begin{aligned}
& { }_{{ }_{1}} \mathbf{L}_{d=1}^{n}{ }_{d} \mathbb{E}_{q}(\mathcal{Z})=\left\{w \in \mathbb{Q}: \cap \mathcal{H}_{\mathbb{E}_{q}}^{\mathcal{R}_{1}}(w) \subseteq \mathcal{Z} \text { or } \cap \mathcal{H}_{\mathbb{E}_{q}}^{\mathcal{R}_{2}}(w) \subseteq \mathcal{Z}\right. \\
& \text { or } \left.\cdots \text { or } \cap \mathcal{H}_{\mathbb{E}_{q}}^{\mathcal{R}_{n}}(w) \subseteq \mathcal{Z}\right\} \text {, } \\
& { }_{1} \mathbf{U}_{d=1}^{n}{ }_{d} \mathbb{E}_{q}(\mathcal{Z})=\left\{w \in \mathbb{Q}:\left[\cap \mathcal{H}_{\mathbb{E}_{q}}^{\mathcal{R}_{1}}(w)\right] \cap \mathcal{Z} \neq \varnothing\right. \\
& \text { and }\left[\cap \mathcal{H}_{\mathbb{E}_{q}}^{\mathcal{R}_{2}}(w)\right] \cap \mathcal{Z} \neq \varnothing \\
& \text { and } \left.\cdots \text { and }\left[\cap \mathcal{H}_{\mathbb{E}_{q}}^{\mathcal{R}_{n}}(w)\right] \cap \mathcal{Z} \neq \varnothing\right\} \text {. }
\end{aligned}
$$

If ${ }_{1} \mathbf{L}_{\sum_{d=1}^{n} d \mathbb{E}_{q}}(\mathscr{E}) \neq{ }_{1} \mathbf{U}_{\sum_{d=1}^{n} d \mathbb{E}_{q}}(\mathscr{X})$, then $\mathscr{E}$ is said to be the first kind of $q$-covering multigranulation rough set (briefly, $1-q$ MGCRS), else it is definable.

Style 2

The 2-MCLA ${ }_{2} \mathbf{L}_{\sum_{d=1}^{n} d \mathbb{E}_{q}}(\mathscr{Z})$ and the 2-MCUA ${ }_{2} \mathbf{L}_{\sum_{d=1 d}^{n} \mathbb{E}_{q}}$ $(\mathscr{Z})$ are seen as follows:

$$
\begin{aligned}
&{ }_{2} \mathbf{L}_{d=1}^{n}(\mathcal{Z})=\left\{w \in \mathbb{Q}: \cup \mathcal{H}_{\mathbb{E}_{q}}^{\mathcal{R}_{1}}(w) \subseteq \mathcal{Z} \text { or } \cup \mathcal{H}_{\mathbb{E}_{q}}^{\mathcal{R}_{2}}(w) \subseteq \mathcal{Z}\right. \\
&\text { or } \left.\cdots \text { or } \cup \mathcal{H}_{\mathbb{E}_{q}}^{\mathcal{R}_{n}}(w) \subseteq \mathcal{Z}\right\}, \\
& \sum_{d=1} \mathbf{U}_{d} \mathbb{E}_{q}(\mathcal{Z})=\left\{w \in \mathbb{Q}:\left[\cup \mathcal{H}_{\mathbb{E}_{q}}^{\mathcal{R}_{1}}(w)\right] \cap \mathcal{Z} \neq \varnothing\right. \\
& \text { and }\left[\cup \mathcal{H}_{\mathbb{E}_{q}}^{\mathcal{R}_{2}}(w)\right] \cap \mathcal{Z} \neq \varnothing \\
&\text { and } \left.\cdots \text { and }\left[\cup \mathcal{H}_{\mathbb{E}_{q}}^{\mathcal{R}_{n}}(w)\right] \cap \mathcal{Z} \neq \varnothing\right\} .
\end{aligned}
$$

If ${ }_{2} \mathbf{L}_{\sum_{d=1}^{n} d \mathbb{E}_{q}}(\mathscr{E}) \neq{ }_{2} \mathbf{U}_{\sum_{d=1}^{n} d \mathbb{E}_{q}}(\mathscr{X})$, then $\mathscr{E}$ is said to be the second kind of $q$-covering multigranulation rough set (briefly, 2-qMGCRS), else it is definable.
Style 3

The 3-MCLA ${ }_{3} \mathbf{L}_{\sum_{d=1}^{n} \mathbb{E}_{q}}(\mathscr{Z})$ and the 3-MCUA ${ }_{3} \mathbf{U}_{\sum_{d=1}^{n} d \mathbb{E}_{q}}$ $(\mathscr{Z})$ are seen as follows:

$$
\begin{aligned}
& { }_{3} \mathbf{L}_{d=1}^{n}{ }_{d} \mathbb{E}_{q}(\mathscr{Z})=\left\{w \in \mathbb{Q}: \cap \mathscr{D}_{\mathbb{E}_{q}}^{\mathscr{R}_{1}}(w) \subseteq \mathscr{Z} \text { or } \cap \mathscr{D}_{\mathbb{E}_{q}}^{\mathscr{R}_{2}}(w) \subseteq \mathscr{Z}\right. \\
& \text { or } \left.\cdots \text { or } \cap \mathscr{D}_{\mathbb{E}_{q}}^{\mathscr{R}_{n}}(w) \subseteq \mathscr{Z}\right\} \text {, } \\
& { }_{3} \sum_{d=1}^{n}{ }_{d} \mathbb{E}_{q}(\mathscr{Z})=\left\{w \in \mathbb{Q}:\left[\cap \mathscr{D}_{\mathbb{E}_{q}}^{\mathscr{R}_{1}}(w)\right] \cap \mathscr{Z} \neq \varnothing\right. \\
& \text { and }\left[\cap \mathscr{D}_{\mathbb{E}_{q}}^{\mathscr{R}_{2}}(w)\right] \cap \mathscr{Z} \neq \varnothing \\
& \text { and } \left.\cdots \text { and }\left[\cap \mathscr{D}_{\mathbb{E}_{q}}^{\mathscr{R}_{n}}(w)\right] \cap \mathscr{E} \neq \varnothing\right\} \text {. }
\end{aligned}
$$

If ${ }_{3} \mathbf{L}_{\sum_{d=1 d}^{n} \mathbb{E}_{q}}(\mathscr{Z}) \neq{ }_{3} \mathbf{U}_{\sum_{d=1 d}^{n} \mathbb{E}_{q}}(\mathscr{Z})$, then $\mathscr{E}$ is said to be the third kind of $q$-covering multigranulation rough set (briefly, 3-qMGCRS), else it is definable.

Style 4

The 4-MCLA ${ }_{4} \mathbf{L}_{\sum_{d=1}^{n} \mathbb{E}_{q}}(\mathscr{Z})$ and the 4-MCUA ${ }_{4} \mathbf{U}_{\sum_{d=1 d}^{n} \mathbb{E}_{q}}$ $(\mathscr{Z})$ are seen as follows:

$$
\begin{aligned}
& { }_{4} \sum_{d=1}{ }_{d} \mathbb{E}_{q}(\mathscr{Z})=\left\{w \in \mathbb{Q}: \cup \mathscr{D}_{\mathbb{E}_{q}}^{\mathscr{R}_{1}}(w) \subseteq \mathscr{Z} \text { or } \cup \mathscr{D}_{\mathbb{E}_{q}}^{\mathscr{R}_{2}}(w) \subseteq \mathscr{Z}\right. \\
& \text { or } \left.\cdots \text { or } \cup \mathscr{D}_{\mathbb{E}_{q}}^{\mathscr{R}_{n}}(w) \subseteq \mathscr{Z}\right\} \text {, } \\
& { }_{4} \mathbf{U}_{d=1}^{n}{ }_{d} \mathbb{E}_{q}(\mathscr{E})=\left\{w \in \mathbb{Q}:\left[\cup \mathscr{D}_{\mathbb{E}_{q}}^{\mathscr{R}_{1}}(w)\right] \cap \mathscr{Z} \neq \varnothing\right. \\
& \text { and }\left[\cup \mathscr{D}_{\mathbb{E}_{q}}^{\mathscr{R}_{2}}(w)\right] \cap \mathscr{Z} \neq \varnothing \\
& \text { and } \left.\cdots \text { and }\left[\cup \mathscr{D}_{\mathbb{E}_{q}}^{\mathscr{R}_{n}}(w)\right] \cap \mathscr{Z} \neq \varnothing\right\} \text {. }
\end{aligned}
$$

If ${ }_{4} \mathbf{L}_{\sum_{d=1}^{n} d \mathbb{E}_{q}}(\mathscr{Z}) \neq{ }_{4} \mathbf{U}_{\sum_{d=1 d}^{n} \mathbb{E}_{q}}(\mathscr{Z})$, then $\mathscr{Z}$ is said to be the fourth kind of $q$-covering multigranulation rough set (briefly, 4-qMGCRS), else it is definable.

Example 3. Consider $\left(\mathbb{Q}, \mathfrak{R}, \mathbb{E}_{q}\right)$ is a $\mathrm{M} \mathbb{E}_{q} \mathrm{CAS}, \mathbb{Q}=\left\{k_{1}, k_{2}\right.$, $\left.k_{3}, k_{4}\right\}$ and $\mathfrak{R}=\left\{\mathscr{R}_{1}, \mathscr{R}_{2}\right\}$, where $\mathscr{R}_{1}=\left\{\left(k_{1}, k_{4}\right),\left(k_{2}, \mathrm{k}_{2}\right)\right.$, $\left.\left(k_{2}, k_{3}\right),\left(k_{3}, k_{2}\right),\left(k_{4}, k_{1}\right),\left(k_{4}, k_{3}\right)\right\}$ and $\mathscr{R}_{2}=\left\{\left(k_{1}, k_{1}\right),\left(k_{1}\right.\right.$, $\left.\left.k_{2}\right),\left(k_{2}, k_{3}\right),\left(k_{2}, k_{4}\right),\left(k_{3}, k_{1}\right),\left(k_{4}, k_{1}\right)\right\}$. Take $\mathscr{Z}=\left\{k_{1}, k_{3}\right\}$; then, we have the presented outcomes:

$\left(1_{\mathrm{r}}\right)_{1} \mathbf{L}_{\sum_{d=1}^{2} \mathbb{E}_{\mathbf{r}}}(\mathcal{Z})=\left\{k_{1}, k_{3}\right\},{ }_{1} \mathbf{U}_{\sum_{d=1}^{2} \mathbb{E}_{\mathbf{r}}}(\mathcal{Z})=\left\{k_{1}, k_{3}\right\}$

$\left(1_{1}\right)_{1} \mathbf{L}_{\sum_{d=1 d}^{2} \mathbb{E}_{1}}(\mathcal{Z})=\left\{k_{1}\right\},{ }_{1} \mathbf{U}_{\sum_{d=1}^{2} \mathbb{E}_{1}}(\mathcal{Z})=\left\{k_{1}, k_{3}\right\}$.

$$
\left.(2)_{\mathbf{r}}\right)_{2} \mathbf{L}_{\sum_{d=1 d}^{2} \mathbb{E}_{\mathbf{r}}}(\mathcal{Z})=\left\{k_{1}\right\},{ }_{2} \mathbf{U}_{\sum_{d=1 d}^{2} \mathbb{E}_{\mathbf{r}}}(\mathcal{Z})=\left\{k_{1}, k_{3}\right\}
$$

$\left(2_{1}\right)_{2} \mathbf{L}_{\sum_{d=1}^{2} d \mathbb{E}_{1}}(\mathcal{Z})=\left\{k_{1}\right\},{ }_{2} \mathbf{U}_{\sum_{d=1 d}^{2} \mathbb{E}_{1}}(\mathcal{Z})=\left\{k_{1}, k_{3}\right\}$.

$\left(3_{\mathrm{r}}\right)_{3} \mathbf{L}_{\sum_{d=1}^{2}{ }_{d} \mathbb{E}_{\mathbf{r}}}(\mathcal{Z})=\left\{k_{1}, k_{3}\right\},{ }_{3} \mathbf{U}_{\sum_{d=1 d}^{2} \mathbb{E}_{\mathbf{r}}}(\mathcal{Z})=\left\{k_{1}, k_{2}, k_{3}\right\}$

$\left(3_{1}\right)_{3} \mathbf{L}_{\sum_{d=1 d}^{2} \mathbb{E}_{1}}(\mathcal{Z})=\left\{k_{1}\right\},{ }_{3} \mathbf{U}_{\sum_{d=1 d}^{2} \mathbb{E}_{1}}(\mathcal{Z})=\left\{k_{1}, k_{3}\right\}$. 
$\left(4_{\mathbf{r}}\right)_{4} \mathbf{L}_{\sum_{d=1}^{2} \mathbb{E}_{\mathbf{r}}}(\mathcal{Z})=\left\{k_{1}\right\},{ }_{4} \mathbf{U}_{\sum_{d=1 d}^{2} \mathbb{E}_{\mathbf{r}}}(\mathcal{Z})=\left\{k_{1}, k_{2}, k_{3}\right\}$

$\left(4_{1}\right)_{4} \mathbf{L}_{\sum_{d=1}^{2} d \mathbb{E}_{1}}(\mathcal{Z})=\left\{k_{1}\right\},{ }_{4} \mathbf{U}_{\sum_{d=1}^{2} d \mathbb{E}_{1}}(\mathcal{Z})=\left\{k_{1}, k_{3}\right\}$.

Theorem 14. Suppose that $\left(\mathbb{Q}, \Re, \mathbb{E}_{q}\right)$ is a $M \mathbb{E}_{q} C A S$. For any $\mathscr{Z} \subseteq \mathbb{Q}$, we get the following properties:

(1) ${ }_{1} \mathbf{L}_{\sum_{d=1 d}^{n} \mathbb{E}_{q}}\left(\mathscr{Z}^{c}\right)=\left[{ }_{1} \mathbf{U}_{\sum_{d=1 d}^{n} \mathbb{E}_{q}}(\mathscr{Z})\right]^{c},{ }_{1} \mathbf{U}_{\sum_{d=1 d}^{n} \mathbb{E}_{q}}\left(\mathscr{Z}^{c}\right)=$ $\left[{ }_{1} \mathbf{L}_{\sum_{d=1}^{n} d \mathbb{E}_{q}}(\mathscr{Z})\right]^{c}$

(2) ${ }_{2} \mathbf{L}_{\sum_{d=1 d}^{n} \mathbb{E}_{q}}\left(\mathscr{Z}^{c}\right)=\left[{ }_{2} \mathbf{U}_{\sum_{d=1 d}^{n} \mathbb{E}_{q}}(\mathscr{Z})\right]^{c},{ }_{2} \mathbf{U}_{\sum_{d=1 d}^{n} \mathbb{E}_{q}}\left(\mathscr{Z}^{c}\right)=$ $\left[{ }_{2} \mathbf{L}_{\sum_{d=1 d}^{n} \mathbb{E}_{q}}(\mathscr{Z})\right]^{c}$

(3) ${ }_{3} \mathbf{L}_{\sum_{d=1}^{n} \mathbb{E}_{q}}\left(\mathscr{Z}^{c}\right)=\left[{ }_{3} \mathbf{U}_{\sum_{d=1}^{n} \mathbb{E}_{q}}(\mathscr{Z})\right]^{c},{ }_{3} \mathbf{U}_{\sum_{d=1 d}^{n} \mathbb{E}_{q}}\left(\mathscr{X}^{c}\right)=$ $\left[{ }_{3} \mathbf{L}_{\sum_{d=1}^{n} d} \mathbb{E}_{q}(\mathscr{Z})\right]^{c}$

(4) ${ }_{4} \mathbf{L}_{\sum_{d=1 d}^{n} \mathbb{E}_{q}}\left(\mathscr{Z}^{c}\right)=\left[{ }_{4} \mathbf{U}_{\sum_{d=1 d}^{n} \mathbb{E}_{q}}(\mathscr{Z})\right]^{c},{ }_{4} \mathbf{U}_{\sum_{d=1 d}^{n} \mathbb{E}_{q}}\left(\mathscr{X}^{c}\right)=$ $\left[{ }_{4} \mathbf{L}_{\sum_{d=1}^{n} d \mathbb{E}_{q}}(\mathscr{Z})\right]^{c}$

Proof. Here, we want to set (1) only.

(1)

$$
\begin{aligned}
& { }_{1} \mathbf{L}_{d=1}^{n}{ }_{d} \mathbb{E}_{q}{ }^{\left(\mathcal{Z}^{c}\right)} \\
& =\left\{w \in \mathbb{Q}: \cap \mathcal{H}_{\mathbb{E}_{q}}^{\mathcal{R}_{1}}(w) \subseteq \mathcal{Z}^{c} \text { or } \cap \mathcal{H}_{\mathbb{E}_{q}}^{\mathcal{R}_{2}}(w) \subseteq \mathcal{Z}^{c}\right. \\
& \text { or } \left.\cdots \text { or } \cap \mathcal{H}_{\mathbb{E}_{q}}^{\mathcal{R}_{n}}(w) \subseteq \mathcal{Z}^{c}\right\} \\
& =\left\{w \in \mathbb{Q}: \cap \mathcal{H}_{\mathbb{E}_{q}^{1}}^{\mathcal{R}_{1}}(w) \cap \mathcal{Z}=\varnothing \text { or } \cap \mathcal{H}_{\mathbb{E}_{q}}^{\mathcal{R}_{2}}(w) \cap \mathcal{Z}=\varnothing\right. \\
& \text { or } \left.\cdots \text { or } \cap \mathcal{H}_{\mathbb{E}_{q}}^{\mathcal{R}_{n}}(w) \cap \mathcal{Z}=\varnothing\right\} \\
& =\left\{w \in \mathbb{Q}: \cap \mathcal{H}_{\mathbb{E}_{q}}^{\mathscr{R}_{1}}(w) \cap \mathcal{Z} \neq \varnothing \text { and } \cap \mathcal{H}_{\mathbb{E}_{q}}^{\mathcal{R}_{2}}(w) \cap \mathcal{Z} \neq \varnothing\right. \\
& \text { and } \left.\cdots \text { and } \cap \mathcal{H}_{\mathbb{E}_{q}}^{\mathcal{R}_{n}}(w) \cap \varnothing\right\}^{c} \\
& =\left[{ }_{1} \mathbf{U}_{\sum_{d=1}^{n}{ }_{d} \mathbb{E}_{q}}(\mathcal{Z})\right]^{c} .
\end{aligned}
$$

Also, it is easy to see ${ }_{1} \mathbf{U}_{\sum_{d=1}^{n} d \mathbb{E}_{q}}\left(\mathcal{Z}^{c}\right)=\left[{ }_{1} \mathbf{L}_{\sum_{d=1}^{n} d \mathbb{E}_{q}}(\mathcal{Z})\right]^{c}$.

Proposition 15. Suppose that $\left(\mathbb{Q}, \Re, \mathbb{E}_{q}\right)$ is a $M \mathbb{E}_{q} C A S$. For any $\mathscr{Z} \subseteq \mathbb{Q}$, we get the following properties:

(1) ${ }_{1} \mathbf{L}_{\sum_{d=1 d}^{n} \mathbb{E}_{q}}\left({ }_{1} \mathbf{L}_{\sum_{d=1}^{n} d \mathbb{E}_{q}}(\mathscr{Z})\right)={ }_{1} \mathbf{L}_{\sum_{d=1}^{n} d \mathbb{E}_{q}}(\mathscr{Z}),{ }_{1} \mathbf{U}_{\sum_{d=1}^{n} d \mathbb{E}_{q}}$ $\left({ }_{1} \mathbf{U}_{\sum_{d=1}^{n} d \mathbb{E}_{q}}(\mathscr{Z})\right)={ }_{1} \mathbf{U}_{\sum_{d=1}^{n} d \mathbb{E}_{q}}(\mathscr{Z})$

(2) ${ }_{2} \mathbf{L}_{\sum_{d=1 d}^{n} \mathbb{E}_{q}}\left({ }_{2} \mathbf{L}_{\sum_{d=1}^{n} d \mathbb{E}_{q}}(\mathscr{X})\right)={ }_{2} \mathbf{L}_{\sum_{d=1}^{n} d \mathbb{E}_{q}}(\mathscr{Z}),{ }_{2} \mathbf{U}_{\sum_{d=1 d}^{n} \mathbb{E}_{q}}$ $\left({ }_{2} \mathbf{U}_{\sum_{d=1}^{n} d \mathbb{E}_{q}}(\mathscr{Z})\right)={ }_{2} \mathbf{U}_{\sum_{d=1}^{n} d \mathbb{E}_{q}}(\mathscr{Z})$

(3) ${ }_{3} \mathbf{L}_{\sum_{d=1}^{n} d \mathbb{E}_{q}}\left({ }_{3} \mathbf{L}_{\sum_{d=1}^{n} d \mathbb{E}_{q}}(\mathscr{Z})\right)={ }_{3} \mathbf{L}_{\sum_{d=1}^{n} d \mathbb{E}_{q}}(\mathscr{Z}),{ }_{3} \mathbf{U}_{\sum_{d=1 d}^{n} \mathbb{E}_{q}}$ $\left({ }_{3} \mathbf{U}_{\sum_{d=1}^{n} d} \mathbb{E}_{q}(\mathscr{Z})\right)={ }_{3} \mathbf{U}_{\sum_{d=1}^{n} d \mathbb{E}_{q}}(\mathscr{E})$
Proof. Here, we want to set (1) only.

(1) It is obvious that ${ }_{1} \mathbf{L}_{\sum_{d=1}^{n} d \mathbb{E}_{q}}\left({ }_{1} \mathbf{L}_{\sum_{d=1 d}^{n} \mathbb{E}_{q}}(\mathscr{Z})\right) \subseteq$ ${ }_{1} \mathbf{L}_{\sum_{d=1}^{n} \mathbb{E}_{q}}(\mathscr{E})$. On the other hand, we have ${ }_{1} \mathbf{L}_{\sum_{d=1 d}^{n} \mathbb{E}_{q}}(\mathscr{E})={ }_{1} \mathbf{L}_{1} \mathbb{E}_{q}(\mathscr{Z}) \cup{ }_{1} \mathbf{L}_{2} \mathbb{E}_{q}(\mathscr{E}) \cup \cdots \cup{ }_{1} \mathbf{L}_{n} \mathbb{E}_{q}(\mathscr{E})$. Thus, we get that

$$
\begin{aligned}
& { }_{1} \mathbf{L}_{d=1}^{n}{ }_{d} \mathbb{E}_{q}\left({ }_{1} \mathbf{L}_{\sum_{d=1}^{n}{ }_{d} \mathbb{E}_{q}}{ }^{(\mathscr{E})}\right) \\
& ={ }_{1} \mathbf{L}_{1} \mathbb{E}_{q}\left({ }_{1} \mathbf{L}_{\sum_{d=1}^{n}{ }_{d} \mathbb{E}_{q}}(\mathscr{Z})\right) \cup{ }_{1} \mathbf{L}_{2} \mathbb{E}_{q}\left({ }_{{ }_{1}} \mathbf{L}_{\sum_{d=1}^{n}}{ }_{d} \mathbb{E}_{q}(\mathscr{Z})\right) \\
& \cup \cdots \cup_{1} \mathbf{L}_{n} \mathbb{E}_{q}\left({ }_{1} \mathbf{L}_{d=1} \sum_{d} \mathbb{E}_{q}(\mathscr{Z})\right) \\
& ={ }_{1} \mathbf{L}_{1} \mathbb{E}_{q}\left({ }_{1} \mathbf{L}_{1} \mathbb{E}_{q}(\mathscr{Z}) \cup{ }_{1} \mathbf{L}_{2} \mathbb{E}_{q}(\mathscr{Z}) \cup \cdots \cup \cup_{1} \mathbf{L}_{n} \mathbb{E}_{q}(\mathscr{Z})(\mathscr{Z})\right) \\
& \cup{ }_{1} \mathbf{L}_{2} \mathbb{E}_{q}\left({ }_{1} \mathbf{L}_{1} \mathbb{E}_{q}(\mathscr{Z}) \cup{ }_{1} \mathbf{L}_{2} \mathbb{E}_{q}(\mathscr{Z}) \cup \cdots \cup_{1} \mathbf{L}_{n} \mathbb{E}_{q}(\mathscr{Z})(\mathscr{Z})\right) \\
& \cup \cdots \cup_{1} \mathbf{L}_{n} \mathbb{E}_{q}\left({ }_{1} \mathbf{L}_{1} \mathbb{E}_{q}(\mathscr{Z}) \cup{ }_{1} \mathbf{L}_{2} \mathbb{E}_{q}(\mathscr{X}) \cup \cdots \cup_{1} \mathbf{L}_{n} \mathbb{E}_{q}(\mathscr{Z})(\mathscr{X})\right) \\
& \supseteq{ }_{1} \mathbf{L}_{1} \mathbb{E}_{q}\left({ }_{1} \mathbf{L}_{1} \mathbb{E}_{q}(\mathscr{Z})\right) \cup{ }_{1} \mathbf{L}_{2} \mathbb{E}_{q}\left({ }_{1} \mathbf{L}_{2} \mathbb{E}_{q}(\mathscr{X})\right) \\
& \cup_{11} \mathbf{L}_{n} \mathbb{E}_{q}\left({ }_{1} \mathbf{L}_{n} \mathbb{E}_{q}(\mathscr{Z})\right) \\
& ={ }_{1} \mathbf{L}_{1} \mathbb{E}_{q}(\mathscr{Z}) \cup{ }_{1} \mathbf{L}_{2} \mathbb{E}_{q}(\mathscr{Z}) \cup \cdots \cup_{1} \mathbf{L}_{n} \mathbb{E}_{q}(\mathscr{Z})={ }_{1} \mathbf{L}_{d=1} \sum_{d} \mathbb{E}_{q}(\mathscr{Z})
\end{aligned}
$$

Also, it is clear that ${ }_{1} \mathbf{U}_{\sum_{d=1 d}^{n} \mathbb{E}_{q}}\left({ }_{1} \mathbf{U}_{\sum_{d=1 d}^{n} \mathbb{E}_{q}}(\mathcal{Z})\right) \subseteq_{1}$ $\mathbf{U}_{\sum_{d=1 d}^{n} \mathbb{E}_{q}}(\mathcal{Z})$. Consequently, we have ${ }_{1} \mathbf{U}_{\sum_{d=1 d}^{n} \mathbb{E}_{q}}(\mathcal{Z})={ }_{1} \mathbf{U}_{1} \mathbb{E}_{q}$ $(\mathcal{Z}) \cap{ }_{1} \mathbf{U}_{2} \mathbb{E}_{q}(\mathcal{Z}) \cap \cdots \cap{ }_{1} \mathbf{U}_{n} \mathbb{E}_{q}(\mathcal{Z})$. So, we have

$$
\begin{aligned}
& { }_{1} \mathbf{U}_{d=1}^{n}{ }_{d} \mathbb{E}_{q}\left({ }^{1} \mathbf{U}_{d=1}^{n}{ }_{d} \mathbb{E}_{q}(\mathcal{Z})\right) \\
& ={ }_{1} \mathbf{U}_{1} \mathbb{E}_{q}\left({ }_{{ }_{1}} \mathbf{U}_{d=1}{ }_{d} \mathbb{E}_{q}(\mathcal{Z})\right) \cap{ }_{1} \mathbf{U}_{2} \mathbb{E}_{q}\left({ }_{1} \mathbf{U}_{\sum_{d=1}^{n}{ }_{d} \mathbb{E}_{q}}(\mathcal{Z})\right) \\
& \cap \cdots \cap{ }_{1} \mathbf{U}_{n} \mathbb{E}_{q}\left({ }_{1} \mathbf{U}_{\sum_{d=1}^{n}{ }_{d} \mathbb{E}_{q}}(\mathcal{Z})\right) \\
& ={ }_{1} \mathbf{U}_{1 \mathbb{E}_{q}}\left({ }_{1} \mathbf{U}_{1} \mathbb{E}_{q}(\mathcal{Z}) \cap{ }_{1} \mathbf{U}_{2} \mathbb{E}_{q}(\mathcal{Z}) \cap \cdots \cap{ }_{1} \mathbf{U}_{n} \mathbb{E}_{q}(\mathcal{Z})\right) \\
& \cap{ }_{1} \mathbf{U}_{2} \mathbb{E}_{q}\left({ }_{1} \mathbf{U}_{1} \mathbb{E}_{q}(\mathcal{Z}) \cap{ }_{1} \mathbf{U}_{2} \mathbb{E}_{q}(\mathcal{Z}) \cap \cdots \cap{ }_{1} \mathbf{U}_{n} \mathbb{E}_{q}(\mathcal{Z})\right)
\end{aligned}
$$




$$
\begin{aligned}
& \cap \cdots \cap{ }_{1} \mathbf{U}_{n} \mathbb{E}_{q}\left({ }_{1} \mathbf{U}_{1} \mathbb{E}_{q}(\mathcal{Z}) \cap{ }_{1} \mathbf{U}_{2} \mathbb{E}_{q}(\mathcal{Z}) \cap \cdots \cap{ }_{1} \mathbf{U}_{n} \mathbb{E}_{q}(\mathcal{Z})\right) \\
& \supseteq{ }_{1} \mathbf{U}_{1} \mathbb{E}_{q}\left({ }_{1} \mathbf{U}_{1} \mathbb{E}_{q}(\mathcal{Z})\right) \cap{ }_{1} \mathbf{U}_{2} \mathbb{E}_{q}\left({ }_{1} \mathbf{U}_{2} \mathbb{E}_{q}(\mathcal{Z})\right) \cap \cdots \\
& \cap{ }_{1} \mathbf{U}_{n} \mathbb{E}_{q}\left({ }_{1} \mathbf{U}_{n} \mathbb{E}_{q}(\mathcal{Z})\right) \\
& ={ }_{1} \mathbf{U}_{1} \mathbb{E}_{q}(\mathcal{Z}) \cap{ }_{1} \mathbf{U}_{2} \mathbb{E}_{q}(\mathcal{Z}) \cap \cdots \cap{ }_{1} \mathbf{U}_{n} \mathbb{E}_{q}(\mathcal{Z}) \\
& ={ }_{1} \mathbf{U}_{\sum_{n}^{n}}(\mathcal{Z}) .
\end{aligned}
$$

Hence, $\quad{ }_{1} \mathbf{L}_{\sum_{d=1}^{n} d \mathbb{E}_{q}}\left({ }_{1} \mathbf{L}_{\sum_{d=1 d}^{n} \mathbb{E}_{q}}(\mathcal{Z})\right)={ }_{1} \mathbf{L}_{\sum_{d=1}^{n} d \mathbb{E}_{q}}(\mathcal{Z})$ and ${ }_{1} \mathbf{U}_{\sum_{d=1}^{n} d \mathbb{E}_{q}}\left({ }_{1} \mathbf{U}_{\sum_{d=1}^{n} d \mathbb{E}_{q}}(\mathcal{Z})\right)={ }_{1} \mathbf{U}_{\sum_{d=1}^{n} d \mathbb{E}_{q}}(\mathcal{Z})$.

The above Proposition 15 is not true for 4-qMGCRS as in the following example.

Example 4. Consider that $\left(\mathbb{Q}, \mathfrak{R}, \mathbb{E}_{q}\right)$ is a $\mathrm{ME}_{q} \mathrm{CAS}, \mathbb{Q}=\left\{k_{1}\right.$, $\left.k_{2}, k_{3}, k_{4}, k_{5}\right\}$ and $\mathfrak{R}=\left\{\mathscr{R}_{1}, \mathscr{R}_{2}\right\}$, where $\mathscr{R}_{1}=\left\{\left(k_{1}, k_{1}\right),\left(k_{1}\right.\right.$, $\left.\left.k_{3}\right),\left(k_{1}, k_{5}\right),\left(k_{2}, k_{2}\right),\left(k_{3}, k_{3}\right),\left(k_{4}, k_{2}\right),\left(k_{4}, k_{4}\right),\left(k_{4}, k_{5}\right),\left(k_{5}, k_{5}\right)\right\}$ and $\mathscr{R}_{2}=\left\{\left(k_{1}, k_{1}\right),\left(k_{1}, k_{3}\right),\left(k_{2}, k_{2}\right),\left(k_{2}, k_{5}\right),\left(k_{3}, k_{3}\right),\left(k_{4}, k_{3}\right)\right.$, $\left.\left(k_{4}, k_{4}\right),\left(k_{5}, k_{2}\right),\left(k_{5}, k_{5}\right)\right\}$.

Take $\mathscr{Z}_{1}=\left\{k_{1}, k_{3}\right\}$ and $\mathscr{Z}_{2}=\left\{k_{4}, k_{5}\right\}$; then, we have the presented outcomes.

$\left(1_{\mathbf{r}}\right){ }_{4} \mathbf{L}_{\sum_{d=1}^{2} \mathrm{~d} \mathbb{E}_{\mathrm{r}}}\left(\mathcal{Z}_{1}\right)=\left\{k_{1}\right\}$ and ${ }_{4} \mathbf{L}_{\sum_{d=1}^{2} \mathbb{E}_{\mathrm{r}}}\left({ }_{4} \mathbf{U}_{\sum_{d=1}^{2} \mathrm{~d} \mathbb{E}_{\mathrm{r}}}\left(\mathcal{Z}_{1}\right)\right)$ $=\varnothing$. Then, ${ }_{4} \mathbf{L}_{\sum_{d=1}^{2} d \mathbb{E}_{\mathrm{r}}}\left({ }_{4} \mathbf{L}_{\sum_{d=1}^{2} d \mathbb{E}_{\mathrm{r}}}\left(\mathcal{Z}_{1}\right)\right) \neq{ }_{4} \mathbf{L}_{\sum_{d=1}^{2} d \mathbb{E}_{\mathrm{r}}}\left(\mathcal{Z}_{1}\right)$.

$\left(2_{\mathbf{r}}\right)_{4} \mathbf{U}_{\sum_{d=1}^{2} \mathbb{E}_{\mathrm{r}}}\left(\mathcal{Z}_{2}\right)=\left\{k_{2}, k_{3}, k_{4}, k_{5}\right\} \quad$ and $\quad{ }_{4} \mathbf{U}_{\sum_{d=1}^{2} d \mathbb{E}_{\mathbf{r}}}$ $\left({ }_{4} \mathbf{U}_{\sum_{d=1}^{2} d \mathbb{E}_{\mathrm{r}}}\left(\mathcal{Z}_{2}\right)\right)=\mathbb{Q}$. Then, ${ }_{4} \mathbf{U}_{\sum_{d=1 d}^{2} \mathbb{E}_{\mathrm{r}}}\left({ }_{4} \mathbf{U}_{\sum_{d=1}^{2} \mathrm{~d} \mathbb{E}_{\mathrm{r}}}\left(\mathcal{Z}_{2}\right)\right) \neq$ ${ }_{4} \mathbf{U}_{\sum_{d=1 d}^{2} \mathbb{E}_{\mathrm{r}}}\left(\mathcal{Z}_{2}\right)$.

$\left(1_{1}\right){ }_{4} \mathbf{L}_{\sum_{d=1 d}^{2} \mathbb{E}_{1}}\left(\mathcal{Z}_{1}\right)=\left\{k_{3}\right\}$ and ${ }_{4} \mathbf{L}_{\sum_{d=1}^{2} \mathbb{E}_{1}}\left({ }_{4} \mathbf{L}_{\sum_{d=1}^{2} \mathrm{~d} \mathbb{E}_{1}}\left(\mathcal{Z}_{1}\right)\right)$ $=\varnothing$. Then, ${ }_{4} \mathbf{L}_{\sum_{d=1}^{2} d \mathbb{E}_{1}}\left({ }_{4} \mathbf{L}_{\sum_{d=1}^{2} d \mathbb{E}_{1}}\left(\mathcal{Z}_{1}\right)\right) \neq{ }_{4} \mathbf{L}_{\sum_{d=1}^{2} d \mathbb{E}_{1}}\left(\mathcal{Z}_{1}\right)$.

$\left(2_{1}\right)_{4} \mathbf{U}_{\sum_{d=1}^{2} \mathbb{E}_{1}}\left(\mathcal{Z}_{2}\right)=\left\{k_{1}, k_{2}, k_{4}, k_{5}\right\} \quad$ and $\quad{ }_{4} \mathbf{U}_{\sum_{d=1 d}^{2} \mathbb{E}_{1}}$ $\left({ }_{4} \mathbf{U}_{\sum_{d=1 d}^{2} \mathbb{E}_{1}}\left(\mathcal{Z}_{2}\right)\right)=\mathbb{Q}$. Then, $\quad{ }_{4} \mathbf{U}_{\sum_{d=1}^{2} d \mathbb{E}_{1}}\left({ }_{4} \mathbf{U}_{\sum_{d=1 d}^{2} \mathbb{E}_{1}}\left(\mathcal{Z}_{2}\right)\right) \neq$ ${ }_{4} \mathbf{U}_{\sum_{d=1 d}^{2} \mathbb{E}_{1}}\left(\mathcal{Z}_{2}\right)$.

Next, we will establish new properties in Proposition 16. These characteristics are done for 1-qMGCRS, 2-qMGCRS, $3-q \mathrm{MGCRS}$, and 4-qMGCRS, though we demonstrate it in the case of 1-qMGCRS and others are similar.

Proposition 16. Suppose that $\left(\mathbb{Q}, \mathfrak{R}, \mathbb{E}_{q}\right)$ is a $M \mathbb{E}_{q} C A S$. For any $\mathcal{Z}_{1}, \mathcal{Z}_{2} \subseteq \mathbb{Q}$, we get the following properties:

(1) If $\mathcal{Z}_{1} \subseteq \mathcal{Z}_{2}$, then ${ }_{1} \mathbf{L}_{\sum_{d=1}^{n} d \mathbb{E}_{q}}\left(\mathcal{Z}_{1}\right) \subseteq{ }_{1} \mathbf{L}_{\sum_{d=1}^{n} d \mathbb{E}_{q}}\left(\mathcal{Z}_{2}\right)$

(2) If $\mathcal{Z}_{1} \subseteq \mathcal{Z}_{2}$, then ${ }_{1} \mathbf{U}_{\sum_{d=1}^{n} d \mathbb{E}_{q}}\left(\mathcal{Z}_{1}\right) \subseteq{ }_{1} \mathbf{U}_{\sum_{d=1}^{n} d \mathbb{E}_{q}}\left(\mathcal{Z}_{2}\right)$

(3) ${ }_{1} \mathbf{L}_{\sum_{d=1 d}^{n} \mathbb{E}_{q}}\left(\mathcal{Z}_{1} \cap \mathcal{Z}_{2}\right) \subseteq{ }_{1} \mathbf{L}_{\sum_{d=1}^{n} d \mathbb{E}_{q}}\left(\mathcal{Z}_{1}\right) \cap{ }_{1} \mathbf{L}_{\sum_{d=1 d}^{n} \mathbb{E}_{q}}\left(\mathcal{Z}_{2}\right)$

(4) ${ }_{1} \mathbf{L}_{\sum_{d=1 d}^{n} \mathbb{E}_{q}}\left(\mathcal{Z}_{1} \cup \mathcal{Z}_{2}\right) \supseteq{ }_{1} \mathbf{L}_{\sum_{d=1}^{n} d \mathbb{E}_{q}}\left(\mathcal{Z}_{1}\right) \cup{ }_{1} \mathbf{L}_{\sum_{d=1}^{n} d \mathbb{E}_{q}}\left(\mathcal{Z}_{2}\right)$

(5) ${ }_{1} \mathbf{U}_{\sum_{d=1 d}^{n} \mathbb{E}_{q}}\left(\mathcal{Z}_{1} \cup \mathcal{Z}_{2}\right) \supseteq{ }_{1} \mathbf{U}_{\sum_{d=1}^{n} d \mathbb{E}_{q}}\left(\mathcal{Z}_{1}\right) \cup_{1} \mathbf{U}_{\sum_{d=1 d}^{n} \mathbb{E}_{q}}\left(\mathcal{Z}_{2}\right)$

(6) ${ }_{1} \mathbf{U}_{\sum_{d=1 d}^{n} \mathbb{E} \mathbb{E}_{q}}\left(\mathcal{Z}_{1} \cap \mathcal{Z}_{2}\right) \subseteq_{1} \mathbf{U}_{\sum_{d=1 d}^{n} \mathbb{E}_{q}}\left(\mathcal{Z}_{1}\right) \cap_{1} \mathbf{U}_{\sum_{d=1 d}^{n} \mathbb{E}_{q}}\left(\mathcal{Z}_{2}\right)$
Proof. Now, we just need to show (1) and (2).

(1) From Definition 13 and since $\mathscr{Z}_{1} \subseteq \mathscr{Z}_{2}$, then, we obtain the following:

$$
\begin{aligned}
& \sum_{d=1} \mathbf{L}_{d}{ }_{d} \mathbb{E}_{q}^{\left(\mathcal{Z}_{1}\right)} \\
& =\left\{w \in \mathbb{Q}: \cap \mathcal{H}_{\mathbb{E}_{q}}^{\mathcal{R}_{1}}(w) \subseteq \mathcal{Z}_{1} \text { or } \cap \mathcal{H}_{\mathbb{E}_{q}}^{\mathcal{R}_{2}}(w) \subseteq \mathcal{Z}_{1}\right. \\
& \text { or } \left.\cdots \text { or } \cap \mathcal{H}_{\mathbb{E}_{q}}^{\mathcal{R}_{n}}(w) \subseteq \mathcal{Z}_{1}\right\} \\
& \subseteq\left\{w \in \mathbb{Q}: \cap \mathcal{H}_{\mathbb{E}_{q}}^{\mathscr{R}_{1}}(w) \subseteq \mathcal{Z}_{2} \text { or } \cap \mathcal{H}_{\mathbb{E}_{q}}^{\mathcal{R}_{2}}(w)\right. \\
& \left.\subseteq \mathcal{Z}_{2} \text { or } \cdots \text { or } \cap \mathcal{H}_{\mathbb{E}_{q}}^{\mathcal{R}_{n}}(w) \subseteq \mathcal{Z}_{2}\right\} \\
& ={ }_{1} \mathbf{L}_{d=1}^{n}{ }_{d} \mathbb{E}_{q}\left(\mathcal{Z}_{2}\right)
\end{aligned}
$$

(2) From Definition 13 and since $\mathscr{Z}_{1} \subseteq \mathscr{Z}_{2}$, then, we have the following:

$$
\begin{gathered}
{ }_{1} \mathbf{U}_{d=1} \sum_{d} \mathbb{E}_{q}\left(\mathcal{Z}_{1}\right)=\left\{w \in \mathbb{Q}:\left[\cap \mathcal{H}_{\mathbb{E}_{q}}^{\mathcal{R}_{1}}(w)\right] \cap \mathcal{Z}_{1}\right. \\
\neq \varnothing \text { and }\left[\cap \mathcal{H}_{\mathbb{E}_{q}}^{\mathcal{R}_{2}}(w)\right] \cap \mathcal{Z}_{1} \\
\left.\neq \varnothing \text { and } \cdots \text { and }\left[\cap \mathcal{H}_{\mathbb{E}_{q}}^{\mathcal{R}_{n}}(w)\right] \cap \mathcal{Z}_{1} \neq \varnothing\right\} \\
\subseteq\left\{w \in \mathbb{Q}:\left[\cap \mathcal{H}_{\mathbb{E}_{q}}^{\mathcal{R}_{1}}(w)\right] \cap \mathcal{Z}_{2}\right. \\
\neq \varnothing \text { and }\left[\cap \mathcal{H}_{\mathbb{E}_{q}}^{\mathcal{R}_{2}}(w)\right] \cap \mathcal{Z}_{2} \\
\left.\neq \varnothing \text { and } \cdots \text { and }\left[\cap \mathcal{H}_{\mathbb{E}_{q}}^{\mathcal{R}_{n}}(w)\right] \cap \mathcal{Z}_{2} \neq \varnothing\right\} \\
={ }_{1} \mathbf{U}_{\sum_{d=1}^{n}{ }_{d} \mathbb{E}_{q}}\left(\mathcal{Z}_{2}\right)
\end{gathered}
$$

Example 5. Consider Example 4. Then, we have the following:

$\left(1_{\mathrm{r}}\right)$ Take $\mathscr{Z}_{1}=\left\{k_{2}, k_{3}, k_{4}\right\}$ and $\mathscr{Z}_{2}=\left\{k_{2}, k_{4}, k_{5}\right\}$, then we have ${ }_{1} \mathbf{L}_{\sum_{d=1}^{2} \mathbb{E}_{\mathrm{r}}}\left(\mathscr{Z}_{1}\right)=\left\{k_{2}, k_{3}, k_{4}\right\},{ }_{1} \mathbf{L}_{\sum_{d=1}^{2} d \mathbb{E}_{\mathrm{r}}}\left(\mathscr{X}_{2}\right)=\left\{k_{2}, k_{4}\right.$, $\left.k_{5}\right\}$ and ${ }_{1} \mathbf{L}_{\sum_{d=1 d}^{2} \mathbb{E}_{\mathrm{r}}}\left(\mathscr{E}_{1} \cap \mathscr{E}_{2}\right)=\left\{k_{2}\right\}$. Thus, ${ }_{1} \mathbf{L}_{\sum_{d=1}^{2} d \mathbb{E}_{\mathrm{r}}}\left(\mathscr{E}_{1} \cap\right.$ $\left.\mathscr{Z}_{2}\right) \neq{ }_{1} \mathbf{L}_{\sum_{d=1 d}^{2} \mathbb{E}_{\mathrm{r}}}\left(\mathscr{E}_{1}\right) \cap{ }_{1} \mathbf{L}_{\sum_{d=1 d}^{2} \mathbb{E}_{\mathrm{r}}}\left(\mathscr{Z}_{2}\right)$

$\left(1_{1}\right)$ Take $\mathscr{Z}_{1}=\left\{k_{2}, k_{4}\right\}$ and $\mathscr{Z}_{2}=\left\{k_{2}, k_{5}\right\}$, then we have $\quad{ }_{1} \mathbf{L}_{\sum_{d=1}^{2} d \mathbb{E}_{1}}\left(\mathscr{Z}_{1}\right)=\left\{k_{2}, k_{4}\right\},{ }_{1} \mathbf{L}_{\sum_{d=1 d}^{2} \mathbb{E}_{1}}\left(\mathscr{E}_{2}\right)=\left\{k_{2}, k_{5}\right\}$ and ${ }_{1} \mathbf{L}_{\sum_{d=1 d}^{2} \mathbb{E}_{1}}\left(\mathscr{X}_{1} \cap \mathscr{Z}_{2}\right)=\varnothing$. Thus, ${ }_{1} \mathbf{L}_{\sum_{d=1 d}^{2} \mathbb{E}_{1}}\left(\mathscr{Z}_{1} \cap \mathscr{X}_{2}\right) \neq$ ${ }_{1} \mathbf{L}_{\sum_{d=1 d}^{2} \mathbb{E}_{1}}\left(\mathscr{Z}_{1}\right) \cap{ }_{1} \mathbf{L}_{\sum_{d=1 d}^{2} \mathbb{E}_{1}}\left(\mathscr{Z}_{2}\right)$

$\left(2_{\mathrm{r}}\right)$ Take $\mathscr{Z}_{1}=\left\{k_{1}\right\}$ and $\mathscr{Z}_{2}=\left\{k_{3}\right\}$, then we have ${ }_{1} \mathbf{L}_{\sum_{d=1 d}^{2} \mathbb{E}_{\mathrm{r}}}\left(\mathscr{Z}_{1}\right)=\varnothing,{ }_{1} \mathbf{L}_{\sum_{d=1}^{2} d \mathbb{E}_{\mathrm{r}}}\left(\mathscr{E}_{2}\right)=\left\{k_{3}\right\} \quad$ and $\quad{ }_{1} \mathbf{L}_{\sum_{d=1 d}^{2} \mathbb{E}_{\mathrm{r}}}$ 
$\left(\mathscr{Z}_{1} \cup \mathscr{Z}_{2}\right)=\left\{k_{1}, k_{3}\right\}$. Thus, ${ }_{1} \mathbf{L}_{\sum_{d=1}^{2} \mathbb{E}_{\mathrm{r}}}\left(\mathscr{Z}_{1} \cup \mathscr{Z}_{2}\right) \neq{ }_{1} \mathbf{L}_{\sum_{d=1}^{2} \mathbb{E}_{\mathrm{r}}}$ $\left(\mathscr{Z}_{1}\right) \cup{ }_{1} \mathbf{L}_{\sum_{d=1}^{2} d \mathbb{E}_{\mathrm{r}}}\left(\mathscr{Z}_{2}\right)$

(2) Take $\mathscr{Z}_{1}=\left\{k_{2}\right\}$ and $\mathscr{Z}_{2}=\left\{k_{4}\right\}$, then we have ${ }_{1} \mathbf{L}_{\sum_{d=1 d}^{2} \mathbb{E}_{1}}\left(\mathscr{E}_{1}\right)=\varnothing,{ }_{1} \mathbf{L}_{\sum_{d=1 d}^{2} \mathbb{E}_{1}}\left(\mathscr{E}_{2}\right)=\left\{k_{4}\right\}$ and ${ }_{1} \mathbf{L}_{\sum_{d=1}^{2} d \mathbb{E}_{1}}\left(\mathscr{Z}_{1}\right.$ $\left.\cup \mathscr{E}_{2}\right)=\left\{k_{2}, k_{4}\right\}$. Thus, ${ }_{1} \mathbf{L}_{\sum_{d=1}^{2} d \mathbb{E}_{1}}\left(\mathscr{E}_{1} \cup \mathscr{X}_{2}\right) \neq{ }_{1} \mathbf{L}_{\sum_{d=1 d}^{2} \mathbb{E}_{1}}\left(\mathscr{E}_{1}\right)$ $\cup_{1} \mathbf{L}_{\sum_{d=1}^{2} d \mathbb{E}_{1}}\left(\mathscr{Z}_{2}\right)$

(3. Take $\mathcal{Z}_{1}=\left\{k_{2}\right\}$ and $\mathcal{Z}_{2}=\left\{k_{3}\right\}$, then we have ${ }_{1} \mathbf{U}_{\sum_{d=1}^{2} d \mathbb{E}_{\mathrm{r}}}\left(\mathcal{Z}_{1}\right)=\left\{k_{2}\right\},{ }_{1} \mathbf{U}_{\sum_{d=1 d}^{2} \mathbb{E}_{\mathrm{r}}}\left(\mathcal{Z}_{2}\right)=\left\{k_{1}, k_{3}\right\}$ and ${ }_{1} \mathbf{U}_{\sum_{d=1 d}^{2} \mathbb{E}_{\mathrm{r}}}$ $\left(\mathcal{Z}_{1} \cup \mathcal{Z}_{2}\right)=\left\{k_{1}, k_{2}, k_{3}, k_{4}\right\}$. Thus, ${ }_{1} \mathbf{U}_{\sum_{d=1}^{2} \mathbb{E}_{\mathrm{r}}}\left(\mathcal{Z}_{1} \cup \mathcal{Z}_{2}\right) \neq$ ${ }_{1} \mathbf{U}_{\sum_{d=1}^{2} d \mathbb{E}_{\mathrm{r}}}\left(\mathcal{Z}_{1}\right) \cup{ }_{1} \mathbf{U}_{\sum_{d=1 d}^{2} \mathbb{E}_{\mathrm{r}}}\left(\mathcal{Z}_{2}\right)$

(3) Take $\mathscr{Z}_{1}=\left\{k_{1}\right\}$ and $\mathscr{Z}_{2}=\left\{k_{2}\right\}$, then we have ${ }_{1} \mathbf{U}_{\sum_{d=1 d}^{2} \mathbb{E}_{1}}\left(\mathscr{Z}_{1}\right)=\left\{k_{1}, k_{3}\right\},{ }_{1} \mathbf{U}_{\sum_{d=1 d}^{2} \mathbb{E}_{1}}\left(\mathscr{Z}_{2}\right)=\left\{k_{2}\right\}$ and ${ }_{1} \mathbf{U}_{\sum_{d=1 d}^{2} \mathbb{E}_{1}}$ $\left(\mathscr{Z}_{1} \cup \mathscr{E}_{2}\right)=\left\{k_{1}, k_{2}, k_{3}, k_{5}\right\}$. Thus, ${ }_{1} \mathbf{U}_{\sum_{d=1}^{2} d \mathbb{E}_{1}}\left(\mathscr{E}_{1} \cup \mathscr{X}_{2}\right) \neq$ ${ }_{1} \mathbf{U}_{\sum_{d=1}^{2} d \mathbb{E}_{1}}\left(\mathscr{Z}_{1}\right) \cup{ }_{1} \mathbf{U}_{\sum_{d=1}^{2} d \mathbb{E}_{1}}\left(\mathscr{Z}_{2}\right)$

$\left(4_{r}\right)$ Take $\mathscr{Z}_{1}=\left\{k_{2}, k_{3}\right\}$ and $\mathscr{Z}_{2}=\left\{k_{2}, k_{4}\right\}$, then we have ${ }_{1} \mathbf{U}_{\sum_{d=1}^{2} d \mathbb{E}_{\mathrm{r}}}\left(\mathscr{Z}_{1}\right)=\left\{k_{1}, k_{2}, k_{3}, k_{4}\right\},{ }_{1} \mathbf{U}_{\sum_{d=1}^{2} d \mathbb{E}_{\mathrm{r}}}\left(\mathscr{Z}_{2}\right)=\left\{k_{2}, k_{4}\right\}$ and ${ }_{1} \mathbf{U}_{\sum_{d=1}^{2} d \mathbb{E}_{\mathrm{r}}}\left(\mathscr{X}_{1} \cap \mathscr{Z}_{2}\right)=\left\{k_{2}\right\}$. Thus, ${ }_{1} \mathbf{U}_{\sum_{d=1 d}^{2} \mathbb{E}_{\mathrm{r}}}\left(\mathscr{Z}_{1} \cap \mathscr{Z}_{2}\right)$ $\neq{ }_{1} \mathbf{U}_{\sum_{d=1 d}^{2} \mathbb{E} \mathbb{E}_{\mathrm{r}}}\left(\mathscr{Z}_{1}\right) \cap{ }_{1} \mathbf{U}_{\sum_{d=1 d}^{2} \mathbb{E}_{\mathrm{r}}}\left(\mathscr{E}_{2}\right)$

$\left(4_{1}\right)$ Take $\mathscr{Z}_{1}=\left\{k_{2}, k_{4}\right\}$ and $\mathscr{Z}_{2}=\left\{k_{2}, k_{5}\right\}$, then we have ${ }_{1} \mathbf{U}_{\sum_{d=1 d}^{2} \mathbb{E}_{1}}\left(\mathscr{E}_{1}\right)=\left\{k_{2}, k_{4}, k_{5}\right\},{ }_{1} \mathbf{U}_{\sum_{d=1 d}^{2} \mathbb{E}_{1}}\left(\mathscr{Z}_{2}\right)=\left\{k_{2}, k_{5}\right\}$ and ${ }_{1} \mathbf{U}_{\sum_{d=1}^{2} d \mathbb{E}_{1}}\left(\mathscr{E}_{1} \cap \mathscr{Z}_{2}\right)=\left\{k_{2}\right\}$. Thus, ${ }_{1} \mathbf{U}_{\sum_{d=1 d}^{2} \mathbb{E}_{1}}\left(\mathscr{X}_{1} \cap \mathscr{Z}_{2}\right) \neq$ ${ }_{1} \mathbf{U}_{\sum_{d=1 d}^{2} \mathbb{E}}\left(\mathscr{Z}_{1}\right) \cap{ }_{1} \mathbf{U}_{\sum_{d=1 d}^{2} \mathbb{E}_{1}}$

\section{Relationships among Different Proposed Models}

Next, we present the relationships between the proposed $\mathrm{ME}_{q} \mathrm{CAS}$ models.

By using Definition 13, we obtain the following properties.

Proposition 17. Let $\left(\mathbb{Q}, \mathfrak{R}, \mathbb{E}_{q}\right)$ be a $M \mathbb{E}_{q} C A S$ and $\mathcal{Z} \subseteq \mathbb{Q}$. Then, we have the following results:

(1) ${ }_{4} \mathbf{L}_{\sum_{d=1}^{n} d \mathbb{E}_{q}}(\mathcal{Z}) \subseteq{ }_{2} \mathbf{L}_{\sum_{d=1}^{n} d \mathbb{E}_{q}}(\mathcal{Z}) \subseteq{ }_{1} \mathbf{L}_{\sum_{d=1 d}^{n} \mathbb{E}_{q}}(\mathcal{Z}) \subseteq \mathcal{Z}$

(2) ${ }_{4} \mathbf{L}_{\sum_{d=1 d}^{n} \mathbb{E}_{q}}(\mathcal{Z}) \subseteq{ }_{3} \mathbf{U}_{\sum_{d=1 d}^{n} \mathbb{E}_{q}}(\mathcal{Z}) \subseteq{ }_{1} \mathbf{L}_{\sum_{d=1}^{n} d \mathbb{E}_{q}}(\mathcal{Z}) \subseteq \mathcal{Z}$

(3) $\mathcal{Z} \subseteq{ }_{1} \mathbf{U}_{\sum_{d=1 d}^{n} d \mathbb{E}_{q}}(\mathcal{Z}) \subseteq{ }_{2} \mathbf{U}_{\sum_{d=1 d}^{n} \mathbb{E}_{q}}(\mathcal{Z}) \subseteq{ }_{4} \mathbf{U}_{\sum_{d=1 d}^{n} \mathbb{E}_{q}}(\mathcal{Z})$

(4) $\mathcal{Z} \subseteq{ }_{1} \mathbf{U}_{\sum_{d=1}^{n} d \mathbb{E}_{q}}(\mathcal{Z}) \subseteq{ }_{3} \mathbf{U}_{\sum_{d=1}^{n} d \mathbb{E}_{q}}(\mathcal{Z}) \subseteq{ }_{4} \mathbf{U}_{\sum_{d=1}^{n} d \mathbb{E}_{q}}(\mathcal{Z})$

Remark 18. Let $\left(\mathbb{Q}, \mathfrak{R e}, \mathbb{E}_{q}\right)$ be a $M \mathbb{E}_{q} \mathrm{CAS}$ and $\mathcal{Z} \subseteq \mathbb{Q}$. Then, we have the following results:

(1) ${ }_{2} \mathbf{L}_{\sum_{d=1 d}^{n} \mathbb{E}_{q}}(\mathcal{Z}) \nsubseteq{ }_{3} \mathbf{L}_{\sum_{d=1}^{n} d \mathbb{E}_{q}}(\mathcal{Z})$ and ${ }_{3} \mathbf{L}_{\sum_{d=1}^{n} d \mathbb{E}_{q}}(\mathcal{Z}) \nsubseteq$ ${ }_{2} \mathbf{L}_{\sum_{d=1}^{n} d \mathbb{E}_{q}}(\mathcal{Z})$

(2) ${ }_{2} \mathbf{U}_{\sum_{d=1 d}^{n} \mathbb{E}_{q}}(\mathcal{Z}) \nsubseteq{ }_{3} \mathbf{U}_{\sum_{d=1}^{n} d \mathbb{E}_{q}}(\mathcal{Z})$ and ${ }_{3} \mathbf{U}_{\sum_{d=1 d}^{n} \mathbb{E}_{q}}(\mathcal{Z}) \nsubseteq$ ${ }_{2} \mathbf{U}_{\sum_{d=1}^{n} d \mathbb{E}_{q}}(\mathcal{Z})$

This means that 2-qMGCRS and 3-qMGCRS are independent.
Proposition 19. Let $\left(\mathbb{Q}, \mathfrak{R e}, \mathbb{E}_{q}\right)$ be a $M \mathbb{E}_{q} C A S$ and $\mathcal{Z} \subseteq \mathbb{Q}$. Then, we have the following results:
(1) ${ }_{1} \mathbf{L}_{\sum_{d=1 d}^{n} \mathbb{E}_{q}}(\mathcal{Z})={ }_{2} \mathbf{L}_{\sum_{d=1 d}^{n} \mathbb{E}_{q}}(\mathcal{Z}) \cup{ }_{3} \mathbf{L}_{\sum_{d=1}^{n} d \mathbb{E}_{q}}(\mathcal{Z})$
(2) ${ }_{1} \mathbf{U}_{\sum_{d=1 d}^{n} \mathbb{E}_{q}}(\mathcal{Z})={ }_{2} \mathbf{U}_{\sum_{d=1 d}^{n} \mathbb{E}_{q}}(\mathcal{Z}) \cap{ }_{3} \mathbf{U}_{\sum_{d=1}^{n} d \mathbb{E}_{q}}(\mathcal{Z})$

To illustrate the above characteristic, we give the following example.

Example 6. Consider Example 4 and let $\mathcal{Z}=\left\{k_{1}, k_{2}\right\}$. Then, we have the following outcomes:

(1) For $q=\mathbf{r}$, we have

$$
\begin{aligned}
\sum_{d=1}{ }_{d} \mathbf{L}_{d} \mathbb{E}_{q}(\mathcal{Z}) & ={ }_{2} \mathbf{L}_{2} \sum_{d=1}{ }_{d} \mathbb{E}_{q}(\mathcal{Z}) \\
& =\left\{k_{2}\right\},{ }_{3} \mathbf{L}_{2}(\mathcal{Z}) \\
& ={ }_{4} \mathbf{L}_{d=1} \sum_{d=1}{ }_{d} \mathbb{E}_{q}(\mathcal{Z})=\varnothing, \\
{ }_{1} \mathbf{U}_{q}{ }_{d=1}{ }_{d} \mathbb{E}_{q}(\mathcal{Z}) & ={ }_{2} \mathbf{U}_{2} \sum_{d=1}{ }_{d} \mathbb{E}_{q}(\mathcal{Z})={ }_{3} \mathbf{U}_{2} \sum_{d=1}{ }_{d} \mathbb{E}_{q}(\mathcal{Z}) \\
& =\left\{k_{1}, k_{2}\right\},{ }_{4} \mathbf{U}_{2}(\mathcal{Z})=\left\{k_{1}, k_{2}, k_{3}, k_{5}\right\} \\
\sum_{d=1}{ }_{d} \mathbb{E}_{q} &
\end{aligned}
$$

(2) For $q=1$, we have

$$
\begin{gathered}
\begin{aligned}
\sum_{d=1} \mathbf{L}_{2}{ }_{d} \mathbb{E}_{q} & \sum_{d=1}(\mathcal{Z})={ }_{2} \mathbf{L}_{2} \mathbb{E}_{q}(\mathcal{Z})={ }_{3} \mathbf{L}_{2} \sum_{d=1}{ }_{d} \mathbb{E}_{q}(\mathcal{Z}) \\
=\left\{k_{1}\right\},{ }_{4} \mathbf{L}_{2}(\mathcal{Z})=\varnothing, & \sum_{d=1}{ }_{d} \mathbb{E}_{q}
\end{aligned} \\
\sum_{d=1} \mathbf{U}_{d} \mathbb{E}_{q}(\mathcal{Z})={ }_{2} \mathbf{U}_{2} \sum_{d=1}{ }_{d} \mathbb{E}_{q}(\mathcal{Z})={ }_{3} \mathbf{U}_{2} \sum_{d=1}{ }_{d} \mathbb{E}_{q}(\mathcal{Z}) \\
=\left\{k_{1}, k_{2}, k_{3}, k_{5}\right\},{ }_{4} \mathbf{U}_{\sum_{2}}(\mathcal{Z})=\mathbb{Q} \\
\sum_{d=1}{ }_{d} \mathbb{E}_{q}
\end{gathered}
$$

So, you can find the following:

(1) ${ }_{4} \mathbf{L}_{\sum_{d=1}^{n} d \mathbb{E}_{\mathbf{r}}}(\mathcal{Z}) \subseteq{ }_{2} \mathbf{L}_{\sum_{d=1 d}^{n} \mathbb{E}_{\mathbf{r}}}(\mathcal{Z}) \subseteq{ }_{1} \mathbf{L}_{\sum_{d=1}^{n} d \mathbb{E}_{\mathbf{r}}}(\mathcal{Z}) \subseteq \mathcal{Z}$

(2) ${ }_{4} \mathbf{L}_{\sum_{d=1}^{n} d \mathbb{E}_{\mathbf{r}}}(\mathcal{Z}) \subseteq{ }_{3} \mathbf{L}_{\sum_{d=1 d}^{n} \mathbb{E}_{\mathbf{r}}}(\mathcal{Z}) \subseteq{ }_{1} \mathbf{L}_{\sum_{d=1}^{n} d \mathbb{E}_{\mathbf{r}}}(\mathcal{Z}) \subseteq \mathcal{Z}$

(3) $\mathcal{Z} \subseteq{ }_{1} \mathbf{U}_{\sum_{d=1 d}^{n} \mathbb{E}_{\mathbf{r}}}(\mathcal{Z}) \subseteq{ }_{2} \mathbf{U}_{\sum_{d=1}^{n} d \mathbb{E}_{\mathbf{r}}}(\mathcal{Z}) \subseteq{ }_{4} \mathbf{U}_{\sum_{d=1 d}^{n} \mathbb{E}_{\mathbf{r}}}(\mathcal{Z})$ 
TABLe 1: Table for the lower approximations.

\begin{tabular}{ccccccccc}
\hline & ${ }_{1} \mathbf{L}_{\sum_{d=1}^{n} d \mathbb{E}_{\mathbf{r}}}(\mathcal{Z})$ & ${ }_{2} \mathbf{L}_{\sum_{d=1}^{n} d \mathbb{E}_{\mathbf{r}}}(\mathcal{Z})$ & ${ }_{3} \mathbf{L}_{\sum_{d=1 d}^{n} \mathbb{E}_{\mathbf{r}}}(\mathcal{Z})$ & ${ }_{4} \mathbf{L}_{\sum_{d=1}^{n} d \mathbb{E}_{\mathbf{r}}}(\mathcal{Z})$ & ${ }_{1} \mathbf{L}_{\sum_{d=1}^{n} d \mathbb{E}_{1}}(\mathcal{Z})$ & ${ }_{2} \mathbf{L}_{\sum_{d=1}^{n d} \mathbb{E}_{1}}(\mathcal{Z})$ & ${ }_{3} \mathbf{L}_{\sum_{d=1}^{n} d \mathbb{E}_{1}}(\mathcal{Z})$ & ${ }_{4} \mathbf{L}_{\sum_{d=1}^{n} d \mathbb{E}_{1}}(\mathcal{Z})$ \\
\hline$L_{1}$ & $\sqrt{ }$ & $\sqrt{ }$ & $\sqrt{ }$ & $\sqrt{ }$ & $\sqrt{ }$ & $\sqrt{ }$ & $\sqrt{ }$ & $\sqrt{ }$ \\
$L_{2}$ & $\sqrt{ }$ & $\sqrt{ }$ & $\sqrt{ }$ & $\sqrt{ }$ & $\sqrt{ }$ & $\sqrt{ }$ & $\sqrt{ }$ & $\sqrt{ }$ \\
$L_{3}$ & $\sqrt{ }$ & $\sqrt{ }$ & $\sqrt{ }$ & $\sqrt{ }$ & $\sqrt{ }$ & $\sqrt{ }$ & $\sqrt{ }$ & $\sqrt{ }$ \\
$L_{4}$ & $\times$ & $\times$ & $\times$ & $\times$ & $\times$ & $\times$ & $\times$ & $\times$ \\
$L_{5}$ & $\sqrt{ }$ & $\sqrt{ }$ & $\sqrt{ }$ & $\sqrt{ }$ & $\sqrt{ }$ & $\sqrt{ }$ & $\sqrt{ }$ & $\sqrt{ }$ \\
$L_{6}$ & $\sqrt{ }$ & $\sqrt{ }$ & $\sqrt{ }$ & $\sqrt{ }$ & $\sqrt{ }$ & $\sqrt{ }$ & $\sqrt{ }$ & $\sqrt{ }$ \\
$L_{7}$ & $\sqrt{ }$ & $\sqrt{ }$ & $\sqrt{ }$ & $\sqrt{ }$ & $\sqrt{ }$ & $\sqrt{ }$ & $\sqrt{ }$ & $\sqrt{ }$ \\
$L_{8}$ & $\sqrt{ }$ & $\sqrt{ }$ & $\sqrt{ }$ & $\times$ & $\sqrt{ }$ & $\sqrt{ }$ & $\sqrt{ }$ & $\times$ \\
$L_{9}$ & $\times$ & $\times$ & $\times$ & $\times$ & $\times$ & $\times$ & $\times$ & $\times$ \\
\hline
\end{tabular}

TABLE 2: Table for the upper approximations.

\begin{tabular}{ccccccccc}
\hline & ${ }_{1} \mathbf{U}_{\sum_{d=1}^{n} d \mathbb{E}_{\mathbf{r}}}(\mathcal{Z})$ & ${ }_{2} \mathbf{U}_{\sum_{d=1}^{n} d \mathbb{E}_{\mathbf{r}}}(\mathcal{Z})$ & ${ }_{3} \mathbf{U}_{\sum_{d=1}^{n} d \mathbb{E}_{\mathbf{r}}}(\mathcal{Z})$ & ${ }_{4} \mathbf{U}_{\sum_{d=1 d}^{n} \mathbb{E}_{\mathbf{r}}}(\mathcal{Z})$ & ${ }_{1} \mathbf{U}_{\sum_{d=1 d}^{n} \mathbb{E}_{1}}(\mathcal{Z})$ & ${ }_{2} \mathbf{U}_{\sum_{d=1 d}^{n} \mathbb{E}_{1}}(\mathcal{Z})$ & ${ }_{3} \mathbf{U}_{\sum_{d=1 d}^{n} \mathbb{E}_{1}}(\mathcal{Z})$ & ${ }_{4} \mathbf{U}_{\sum_{d=1}^{n} d \mathbb{E}_{1}}(\mathcal{Z})$ \\
\hline$H_{1}$ & $\sqrt[V]{ }$ & $\sqrt{ }$ & $\sqrt{ }$ & $\sqrt{ }$ & $\sqrt{ }$ & $\sqrt{ }$ & $\sqrt{ }$ & $\sqrt{ }$ \\
$H_{2}$ & $\sqrt{ }$ & $\sqrt{ }$ & $\sqrt{ }$ & $\sqrt{ }$ & $\sqrt{ }$ & $\sqrt{ }$ & $\sqrt{ }$ & $\sqrt{ }$ \\
$H_{3}$ & $\sqrt{ }$ & $\sqrt{ }$ & $\sqrt{ }$ & $\sqrt{ }$ & $\sqrt{ }$ & $\sqrt{ }$ & $\sqrt{ }$ & $\sqrt{ }$ \\
$H_{4}$ & $\times$ & $\times$ & $\times$ & $\times$ & $\times$ & $\times$ & $\times$ & $\times$ \\
$H_{5}$ & $\sqrt{ }$ & $\sqrt{ }$ & $\sqrt{ }$ & $\sqrt{ }$ & $\sqrt{ }$ & $\sqrt{ }$ & $\sqrt{ }$ & $\sqrt{ }$ \\
$H_{6}$ & $\sqrt{ }$ & $\sqrt{ }$ & $\sqrt{ }$ & $\sqrt{ }$ & $\sqrt{ }$ & $\sqrt{ }$ & $\sqrt{ }$ & $\sqrt{ }$ \\
$H_{7}$ & $\sqrt{ }$ & $\sqrt{ }$ & $\sqrt{ }$ & $\sqrt{ }$ & $\sqrt{ }$ & $\sqrt{ }$ & $\sqrt{ }$ & $\sqrt{ }$ \\
$H_{8}$ & $\sqrt{ }$ & $\sqrt{ }$ & $\sqrt{ }$ & $\times$ & $\sqrt{ }$ & $\sqrt{ }$ & $\sqrt{ }$ & $\times$ \\
$H_{9}$ & $\times$ & $\times$ & $\times$ & $\times$ & $\times$ & $\times$ & $\times$ & $\times$ \\
\hline
\end{tabular}

(4) $\mathcal{Z} \subseteq{ }_{1} \mathbf{U}_{\sum_{d=1}^{n} d \mathbb{E}_{\mathbf{r}}}(\mathcal{Z}) \subseteq{ }_{3} \mathbf{U}_{\sum_{d=1 d}^{n} \mathbb{E}_{\mathbf{r}}}(\mathcal{Z}) \subseteq{ }_{4} \mathbf{U}_{\sum_{d=1 d}^{n} \mathbb{E}_{\mathbf{r}}}(\mathcal{Z})$

(5) ${ }_{4} \mathbf{L}_{\sum_{d=1 d}^{n} \mathbb{E}_{1}}(\mathcal{Z}) \subseteq{ }_{2} \mathbf{L}_{\sum_{d=1 d}^{n} \mathbb{E}_{1}}(\mathcal{Z}) \subseteq{ }_{1} \mathbf{L}_{\sum_{d=1 d}^{n} \mathbb{E}_{1}}(\mathcal{Z}) \subseteq \mathcal{Z}$

(6) ${ }_{4} \mathbf{L}_{\sum_{d=1 d}^{n} \mathbb{E}_{1}}(\mathcal{Z}) \subseteq{ }_{3} \mathbf{L}_{\sum_{d=1}^{n} d \mathbb{E}_{1}}(\mathcal{Z}) \subseteq{ }_{1} \mathbf{L}_{\sum_{d=1 d}^{n} \mathbb{E}_{1}}(\mathcal{Z}) \subseteq \mathcal{Z}$

(7) $\mathcal{Z} \subseteq{ }_{1} \mathbf{U}_{\sum_{d=1}^{n} d \mathbb{E}_{1}}(\mathcal{Z}) \subseteq{ }_{2} \mathbf{U}_{\sum_{d=1}^{n} d \mathbb{E}_{1}}(\mathcal{Z}) \subseteq{ }_{4} \mathbf{U}_{\sum_{d=1}^{\mathrm{n} d} \mathbb{E}_{1}}(\mathcal{Z})(\mathcal{Z})$

(8) $\mathcal{Z} \subseteq{ }_{1} \mathbf{U}_{\sum_{d=1}^{n} d \mathbb{E}_{1}}(\mathcal{Z}) \subseteq{ }_{3} \mathbf{U}_{\sum_{d=1 d}^{n} \mathbb{E}_{1}}(\mathcal{Z}) \subseteq{ }_{4} \mathbf{U}_{\sum_{d=1}^{n} d \mathbb{E}_{1}}(\mathcal{Z})$

Tables 1 and 2 show the Pawlak characteristics for the lower and upper approximations which are given in Definition 13 .

\section{Relative Reduction of a $M \mathbb{E}_{q}$ CAS}

This section is aimed at discussing a relative reduction of a pessimistic multigranulation $q$-covering rough sets (briefly, PME $\mathbb{E}_{q} \mathrm{CRS}$ ). First, we give the following couple of definitions.

Definition 20. Let $\left(\mathbb{Q}, \mathfrak{R}, \mathbb{E}_{q}\right)$ be a $\mathrm{M} \mathbb{E}_{q}$ CAS and $\mathfrak{R}=\left\{\mathscr{R}_{1}\right.$, $\left.\mathscr{R}_{2}, \cdots, \mathscr{R}_{S}\right\}, \forall S \in I$. For any $\mathscr{Z} \subseteq \mathbb{Q}$ and $w \in \mathbb{Q}$, define the pessimistic multigranulation $q$-covering lower approximation (briefly, PMGE $\mathbb{E}_{q}$ CLA) $\boldsymbol{L}_{\sum_{d=1}^{n} d_{\mathbb{E}}}^{P}(\mathscr{Z})$ and pessimistic multigranulation $q$-covering lower approximation (briefly, PMGE $\mathbb{E}_{q}$ CLA) $U_{\sum_{d=1 d}^{P} \mathbb{E}_{q}}^{n}(\mathscr{Z})$ as follows:

$$
\begin{aligned}
& \mathbf{L}_{{ }^{n}}^{P}{ }_{d=1}{ }_{d} \mathbb{E}_{q}(\mathscr{Z})=\left\{w \in \mathbb{Q}:(w)_{\mathbb{E}_{q}}^{\mathscr{R}_{1}} \subseteq \mathscr{Z} \text { and }(w)_{\mathbb{E}_{q}}^{\mathscr{R}_{2}} \subseteq \mathscr{Z}\right. \\
&\text { and } \left.\cdots \text { and }(w)_{\mathbb{E}_{q}}^{\mathscr{R}_{n}} \subseteq \mathscr{Z}\right\}, \\
& \sum_{d=1}^{\mathbf{U}_{d}^{P}{ }_{d} \mathbb{E}_{q}}(\mathscr{Z})=\left\{w \in \mathbb{Q}:(w)_{\mathbb{E}_{q}}^{\mathscr{R}_{1}} \cap \mathscr{Z} \neq \varnothing \text { or }(w)_{\mathbb{E}_{q}}^{\mathscr{R}_{2}} \cap \mathscr{Z} \neq \varnothing\right. \\
&\text { or } \left.\cdots \text { or }(w)_{\mathbb{E}_{q}}^{\mathscr{R}_{n}} \cap \mathscr{Z} \neq \varnothing\right\} .
\end{aligned}
$$

Definition 21. Let $\left(\mathbb{Q}, \Re, \mathbb{E}_{q}\right)$ be a $\mathbb{M E}_{q} \mathrm{CAS}$ and $\Re=\left\{\mathscr{R}_{1}\right.$, $\left.\mathscr{R}_{2}, \cdots, \mathscr{R}_{S}\right\}, \forall S \in I$. Suppose that $\mathscr{D}=\left\{\mathscr{D}_{1}, \mathscr{D}_{2}, \cdots, \mathscr{D}_{t}\right\}$ is a decision partition of $\mathbb{Q}$. Then,

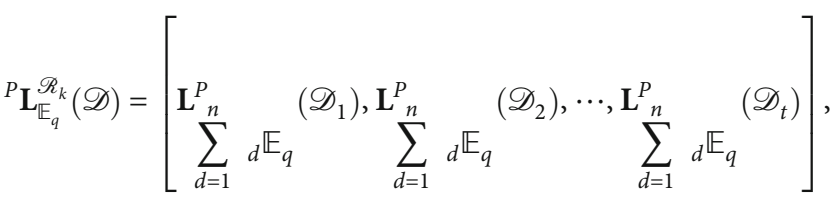


Input: $\left(\mathbb{Q}, \mathfrak{R}, \mathbb{E}_{q}\right)$ with information system.

Output: Reduction of $\mathrm{PM} \mathbb{E}_{q}$ CLA.

1: Calculate ${ }^{P} \mathbf{L}_{\mathbb{E}_{q}}^{\mathscr{R}_{k}}(\mathscr{D})$.

2: Remove $\mathbb{E}_{q}^{\mathscr{R}_{k}}, \mathscr{B}_{q}^{\mathscr{R}_{k}}=\mathbb{E}_{q}^{\mathscr{R}_{i}}-\mathbb{E}_{q}^{\mathscr{R}_{k}}$ and ${ }^{P} \mathbf{L}_{\mathscr{R}_{q}}^{\mathscr{R}_{i-k}}(\mathscr{D})={ }^{P} \mathbf{L}_{\mathscr{R}_{q}}^{\mathscr{R}_{i}}(\mathscr{D})$.

3: Remove a covering in $\mathscr{B}_{q}^{\mathscr{R}_{k}}$ again and get $\mathscr{B}_{\wedge_{q}}^{\mathscr{R}_{i-k}}$. If ${ }^{P} \mathbf{L}_{\mathscr{\mathscr { R }} \wedge_{q}}^{\mathscr{R}_{i-k}}(\mathscr{D}) \neq{ }^{P} \mathbf{L}_{\mathscr{R}_{q}}^{\mathscr{R}_{i}}(\mathscr{D})$, return $\mathscr{B}_{q}^{\mathscr{R}_{k}}$; else, go to Step 2.

4: : Repeat the Steps 2 and 3 for each covering in $\mathbb{E}_{q}^{\mathscr{R}_{i}}$ to get all the relative reduce of the covering family.

Algorithm 1: Algorithm for reduction of $\mathrm{PME}_{q}$ CLA.

TABLE 3: Table for house assessment problem.

\begin{tabular}{lccccc}
\hline & Equally shared area & Color & Price & Surrounding & Purchase options \\
\hline$k_{1}$ & $\{$ Large & $\{$ Good & $\{$ High & $\{$ Very noisy\} & Oppose \\
$k_{2}$ & $\{$ Small, large & $\{$ Excellent & $\{$ Middle, low & $\{$ Quiet, noisy\} & Support \\
$k_{3}$ & $\{$ Small, large & $\{$ Excellent, good & $\{$ Middle, low & Support & Oppose \\
$k_{4}$ & $\{$ Small, ordinary\} & $\{$ Bad & $\{$ High, middle & $\{$ Noisy, very noisy & Oppose \\
$k_{5}$ & $\{$ Small, ordinary\} & $\{$ Bad & $\{$ High, middle & $\{$ Very noisy & Support \\
$k_{6}$ & $\{$ Ordinary, large & $\{$ Excellent, good & $\{$ High, low & $\{$ Quiet, noisy & \\
\hline
\end{tabular}

${ }^{P} \mathbf{U}_{\mathbb{E}_{q}}^{\mathscr{R}_{k}}(\mathscr{D})=\left[\sum_{d=1}^{\mathbf{U}_{d}{ }_{n} \mathbb{E}_{q}}\left(\mathscr{D}_{1}\right), \mathbf{U}_{d=1}^{P} \sum_{{ }_{d}} \mathbb{E}_{q}\left(\mathscr{D}_{2}\right), \cdots, \mathbf{U}_{\sum_{d=1}^{P}{ }_{d} \mathbb{E}_{q}}\left(\mathscr{D}_{t}\right)\right]$.

(i) $\mathscr{B}_{q}^{\mathscr{R}_{k}} \subseteq \mathbb{E}_{q}^{\mathscr{R}_{k}}$ and ${ }^{P} \mathbf{L}_{\mathscr{R}_{q}}^{\mathscr{R}_{k}}(\mathscr{D})={ }^{P} \mathbf{L}_{\mathbb{E}_{q}}^{\mathscr{R}_{k}}(\mathscr{D})$, but ${ }^{P} \mathbf{L}_{\mathscr{B} \wedge_{q}}^{\mathscr{R}_{k}}(\mathscr{D})$ $\neq{ }^{P} \mathbf{L}_{\mathbb{E}_{q}}^{\mathscr{R}_{k}}(\mathscr{D})$, for $\mathscr{B} \wedge_{q}^{\mathscr{R}_{k}} \subseteq \mathscr{B}_{q}^{\mathscr{R}_{k}}$; then, $\mathscr{B}_{q}^{\mathscr{R}_{k}}$ is a $\mathscr{D}$ reduction of $\mathrm{PM} \mathbb{E}_{q} \mathrm{CLA}$

(ii) $\mathscr{B}_{q}^{\mathscr{R}_{k}} \subseteq \mathbb{E}_{q}^{\mathscr{R}_{k}}$ and ${ }^{P} \mathbf{U}_{\mathscr{R}_{q}}^{\mathscr{R}_{k}}(\mathscr{D})={ }^{P} \mathbf{U}_{\mathbb{E}_{q}}^{\mathscr{R}_{k}}(\mathscr{D})$, but ${ }^{P} \mathbf{U}_{\mathscr{R}_{k} \wedge_{q}}^{\mathscr{R}_{k}}$ $(\mathscr{D}) \neq{ }^{P} \mathbf{U}_{\mathbb{E}_{q}}^{\mathscr{R}_{k}}(\mathscr{D})$, for $\mathscr{B} \wedge_{q}^{\mathscr{R}_{k}} \subseteq \mathscr{B}_{q}^{\mathscr{R}_{k}}$, then, $\mathscr{B}_{q}^{\mathscr{R}_{k}}$ is a $\mathscr{D}$ reduction of $\mathrm{PM} \mathbb{E}_{q} \mathrm{CUA}$

We can illustrate the method of reduction as the following Algorithm 1.

Example 7. Presume that $\mathbb{Q}=\left\{k_{1}, k_{2}, \cdots, k_{6}\right\}$ is a set of six houses, $\mathscr{Z}=$ \{equally shared area, color, price, surroundings $\}$ is a set of attributes, and $D=$ \{purchase opinions $\}$ is a set of decisions. The values of equally shared area could be \{large, ordinary, small\}. The values of color could be \{excellent, good, bad\}. The values of price could be \{high, middle, low\}. The values of surroundings could be \{quiet, noisy, very noisy\}. The decision values of purchase opinions could be ssupport, oppose\}, which is randomly chosen from experts. The evaluation results are shown in Table 3.
As for the attribute set $\mathscr{Z}$, the binary relation is obtained as follows $\forall k \in \mathscr{Z}$ :

$$
\mathscr{R}_{k}=\left\{(v, w): \mathscr{F}_{k}(v) \subseteq \mathscr{F}_{k}(w)\right\} .
$$

It is easy to see that the $\mathscr{R}_{k}$ is reflexive and transitive but not symmetric.

If $\mathscr{D}$ is the decision set, then the nonequivalence relation is defined as follows:

$$
\mathscr{R}_{\mathscr{D}}=\left\{(v, w): \mathscr{F}_{\mathscr{D}}(v) \subseteq \mathscr{F}_{\mathscr{D}}(w)\right\} .
$$

Then, we can construct the following two covers:

(i) Right covering (r-cover for short)

$$
\mathscr{C}_{\mathrm{r}}=\left\{w \mathscr{R}_{\mathscr{K}}: \forall \mathscr{K} \in\{\mathscr{Z}, \mathscr{D}\}, \quad w \in \mathbb{Q}, \mathbb{Q}=\bigcup_{w \in \mathbb{Q}} w \mathscr{R}_{\mathscr{K}}\right\}
$$

(ii) Left covering (1-cover for short)

$$
\mathscr{C}_{1}=\left\{\mathscr{R}_{\mathscr{K}} w: \forall \mathscr{K} \in\{\mathscr{Z}, \mathscr{D}\}, \quad w \in \mathbb{Q}, \mathbb{Q}=\bigcup_{w \in \mathbb{Q}} \mathscr{R}_{\mathscr{K}} w\right\}
$$

So, we have the following results:

$$
\begin{aligned}
& \mathscr{C}_{\mathrm{r}}^{\mathscr{R}_{1}}=\left\{\left\{k_{1}, k_{2}, k_{3}\right\},\left\{k_{2}, k_{3}\right\},\left\{k_{4}, k_{5}\right\},\left\{k_{6}\right\}\right\}, \\
& \mathscr{C}_{\mathrm{r}}^{\mathscr{R}_{2}}=\left\{\left\{k_{1}, k_{3}, k_{6}\right\},\left\{k_{2}, k_{3}, k_{6}\right\},\left\{k_{3}, k_{6}\right\},\left\{k_{4}, k_{5}\right\}\right\},
\end{aligned}
$$


TABLE 4: Table for house assessment problem.

\begin{tabular}{lcccccc}
\hline & $k_{1}$ & $k_{2}$ & $k_{3}$ & $k_{4}$ & $k_{5}$ & $k_{6}$ \\
\hline$k \mathscr{C}_{\mathrm{r}}^{\mathfrak{R}_{1}}$ & $\left\{k_{1}, k_{2}, k_{3}\right\}$ & $\left\{k_{2}, k_{3}\right\}$ & $\left\{k_{2}, k_{3}\right\}$ & $\left\{k_{4}, k_{5}\right\}$ & $\left\{k_{4}, k_{5}\right\}$ & $\left\{k_{6}\right\}$ \\
$k \mathscr{C}_{\mathrm{r}}^{\mathfrak{R}_{2}}$ & $\left\{k_{1}, k_{3}, k_{6}\right\}$ & $\left\{k_{2}, k_{3}, k_{6}\right\}$ & $\left\{k_{3}, k_{6}\right\}$ & $\left\{k_{4}, k_{5}\right\}$ & $\left\{k_{4}, k_{5}\right\}$ & $\left\{k_{3}, k_{6}\right\}$ \\
$k \mathscr{C}_{\mathrm{r}}^{\mathfrak{R}_{3}}$ & $\left\{k_{1}, k_{4}, k_{5}, k_{6}\right\}$ & $\left\{k_{2}, k_{3}\right\}$ & $\left\{k_{2}, k_{3}\right\}$ & $\left\{k_{4}, k_{5}\right\}$ & $\left\{k_{4}, k_{5}\right\}$ & $\left\{k_{6}\right\}$ \\
$k \mathscr{C}_{\mathrm{r}}^{\mathfrak{R}_{4}}$ & $\left\{k_{1}, k_{4}, k_{5}\right\}$ & $\left\{k_{2}, k_{6}\right\}$ & $\left\{k_{2}, k_{3}, k_{6}\right\}$ & $\left\{k_{4}\right\}$ & $\left\{k_{1}, k_{4}, k_{5}\right\}$ & $\left\{k_{2}, k_{6}\right\}$ \\
\hline
\end{tabular}

TABLE 5: Table for house assessment problem.

\begin{tabular}{lcccccc}
\hline & $k_{1}$ & $k_{2}$ & $k_{3}$ & $k_{4}$ & $k_{5}$ & $k_{6}$ \\
\hline$k \mathscr{C}_{1}^{\Re_{1}}$ & $\left\{k_{1}\right\}$ & $\left\{k_{1}, k_{2}, k_{3}\right\}$ & $\left\{k_{1}, k_{2}, k_{3}\right\}$ & $\left\{k_{4}, k_{5}\right\}$ & $\left\{k_{4}, k_{5}\right\}$ & $\left\{k_{6}\right\}$ \\
$k \mathscr{C}_{1}^{\mathfrak{R}_{2}}$ & $\left\{k_{1}\right\}$ & $\left\{k_{2}\right\}$ & $\left\{k_{1}, k_{2}, k_{3}, k_{6}\right\}$ & $\left\{k_{4}, k_{5}\right\}$ & $\left\{k_{4}, k_{5}\right\}$ & $\left\{k_{1}, k_{2}, k_{3}, k_{6}\right\}$ \\
$k \mathscr{C}_{1}^{\Re_{3}}$ & $\left\{k_{1}\right\}$ & $\left\{k_{2}, k_{3}\right\}$ & $\left\{k_{2}, k_{3}\right\}$ & $\left\{k_{1}, k_{4}, k_{5}\right\}$ & $\left\{k_{1}, k_{4}, k_{5}\right\}$ & $\left\{k_{1}, k_{6}\right\}$ \\
$k \mathscr{C}_{1}^{\Re_{4}}$ & $\left\{k_{1}, k_{5}\right\}$ & $\left\{k_{2}, k_{3}, k_{6}\right\}$ & $\left\{k_{3}\right\}$ & $\left\{k_{1}, k_{3}, k_{4}, k_{5}\right\}$ & $\left\{k_{1}, k_{5}\right\}$ & $\left\{k_{2}, k_{3}, k_{6}\right\}$ \\
\hline
\end{tabular}

$\mathscr{C}_{\mathrm{r}}^{\mathscr{R}_{3}}=\left\{\left\{k_{1}, k_{4}, k_{5}, k_{6}\right\},\left\{k_{2}, k_{3}\right\},\left\{k_{4}, k_{5}\right\},\left\{k_{6}\right\}\right\}$,

$\mathscr{C}_{\mathrm{r}}^{\mathscr{R}_{4}}=\left\{\left\{k_{1}, k_{4}, k_{5}\right\},\left\{k_{2}, k_{6}\right\},\left\{k_{2}, k_{3}, k_{4}, k_{6}\right\},\left\{k_{4}\right\}\right\}$,

$\mathscr{C}_{\mathrm{r}}^{\mathscr{R}_{\mathscr{P}}}=\left\{\left\{k_{1}, k_{4}, k_{5}\right\},\left\{k_{2}, k_{3}, k_{6}\right\}\right\}$,

$\mathscr{C}_{1}^{\mathscr{R}_{1}}=\left\{\left\{k_{1}\right\},\left\{k_{1}, k_{2}, k_{3}\right\},\left\{k_{4}, k_{5}\right\},\left\{k_{6}\right\}\right\}$,

$\mathscr{C}_{1}^{\mathscr{R}_{2}}=\left\{\left\{k_{1}\right\},\left\{k_{2}\right\},\left\{k_{1}, k_{2}, k_{3}, k_{6}\right\},\left\{k_{4}, k_{5}\right\}\right\}$

$\mathscr{C}_{1}^{\mathscr{R}_{3}}=\left\{\left\{k_{1}\right\},\left\{k_{2}, k_{3}\right\},\left\{k_{1}, k_{4}, k_{5}\right\},\left\{k_{1}, k_{6}\right\}\right\}$,

$\mathscr{C}_{1}^{\mathcal{R}_{4}}=\left\{\left\{k_{1}, k_{5}\right\},\left\{k_{2}, k_{3}, k_{6}\right\},\left\{k_{3}\right\},\left\{k_{1}, k_{3}, k_{4}, k_{5}\right\}\right\}$,

$\mathscr{C}_{1}^{\mathscr{R}_{\mathscr{P}}}=\left\{\left\{k_{1}, k_{4}, k_{5}\right\},\left\{k_{2}, k_{3}, k_{6}\right\}\right\}$.

Thus, we can establish Tables 4 and 5 for the neighborhood of $k$ as follows.

Now, we can apply Algorithm 1 as follows.

Step 1. ${ }^{P} \mathbf{L}_{\mathbb{E}_{\mathrm{r}}}^{\mathscr{R}_{k}}(\mathscr{D})=\left[\mathbf{L}_{\sum_{d=1 d}^{4} \mathbb{E}_{\mathrm{r}}}^{P}\left(\mathscr{D}_{1}\right), \mathbf{L}_{\sum_{d=1 d}^{4} \mathbb{E}_{\mathbf{r}}}^{P}\left(\mathscr{D}_{2}\right)\right]=\left[\left\{k_{4}, k_{5}\right\}\right.$, $\left.\left\{k_{2}, k_{3}, k_{6}\right\}\right]$.

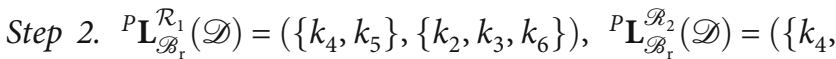
$\left.\left.k_{5}\right\},\left\{k_{2}, k_{3}, k_{6}\right\}\right)$. Therefore, $\mathscr{B}_{\mathrm{r}}^{\mathscr{R}_{k}}=\left\{\mathscr{C}_{\mathrm{r}}^{\mathscr{R}_{3}}, \mathscr{C}_{\mathrm{r}}^{\mathscr{R}_{4}}\right\}$ is a reduction of the PME $\mathbb{E}_{\mathrm{r}} \mathrm{CRS}$.
Also, we can get the following outcomes of the left covering:

$$
\begin{aligned}
& { }^{P} \mathbf{L}_{\mathbb{E}_{1}}^{\mathscr{R}_{k}}(\mathscr{D})=\left[\begin{array}{lr}
\mathbf{L}^{P}{ }_{\sum_{d=1}{ }_{d} \mathbb{E}_{1}}\left(\mathscr{D}_{1}\right), \mathbf{L}^{P}{ }_{{ }_{d}}{ }_{d} \mathbb{E}_{1} & \left(\mathscr{D}_{2}\right)
\end{array}\right]=\left[\left\{k_{1}, k_{5}\right\}, \varnothing\right], \\
& { }^{P} \mathbf{L}_{\mathscr{R}_{1}}^{\mathcal{R}_{1}}(\mathscr{D})=\left(\left\{k_{1}, k_{5}\right\},\left\{k_{2}\right\}\right), \\
& { }^{P} \mathbf{L}_{\mathscr{B}_{1}}^{\mathcal{R}_{2}}(\mathscr{D})=\left(\left\{k_{1}, k_{5}\right\}, \phi\right), \\
& { }^{P} \mathbf{L}_{\mathscr{R}_{1}}^{\mathcal{R}_{3}}(\mathscr{D})=\left(\left\{k_{1}, k_{5}\right\},\left\{k_{6}\right\}\right), \\
& { }^{P} \mathbf{L}_{\mathscr{R}_{1}}^{\mathcal{R}_{4}}(\mathscr{D})=\left(\left\{k_{1}, k_{4}, k_{5}\right\}, \varnothing\right) .
\end{aligned}
$$

Therefore, $\mathscr{B}_{1}^{\mathscr{R}_{k}}=\left\{\mathscr{C}_{1}^{\mathscr{R}_{1}}, \mathscr{C}_{1}^{\mathscr{R}_{3}}, \mathscr{C}_{1}^{\mathscr{R}_{4}}\right\}$ is a reduction of the $\mathrm{PME}_{1} \mathrm{CRS}$.

\section{Conclusion}

In this article, we present a notion called multi- $\mathbb{E}_{q}$-covering approximation space $\left(\mathrm{ME}_{q} \mathrm{CAS}\right)$ by using the concept of $q$ minimal and $q$-maximal descriptions. Based on these notions, we establish four new types of multigranulation covering rough sets, denoted $M \mathbb{E}_{q} \mathrm{CAS}$. We also study the properties of these new models. Further, we put forward a new methodology to make a reduction by the presented work. Then, we demonstrate the reduction method with the help of an illustrative example which shows its effectiveness and reliability. The main differences between our proposed work and the previous one in [39] are that the authors in [39] introduced four types of MGCRSs using the minimal and maximal description based on equivalence relations 
and here we used the notions of right (resp., left) covering rough sets to investigate four kinds of multigranulation right (resp., left) covering rough sets using the right (resp., left) minimal and right (resp., left) maximal description induced by binary relations. In further research, we hope to use this approach in fuzzy rough covering-based fuzzy neighborhoods [49], fuzzy soft covering-based rough sets [50], and soft fuzzy covering-based rough sets [51].

\section{Data Availability}

No data were used to support this study.

\section{Conflicts of Interest}

The authors declare that they have no conflicts of interest.

\section{Funding}

The second author is responsible for funding this work.

\section{References}

[1] Z. Pawlak, "Rough sets," International Journal of Computer and Information Science, vol. 11, no. 5, pp. 341-356, 1982.

[2] Z. Pawlak, "Rough concept analysis," Bulletin of the Polish Academy of Sciences Mathematics, vol. 33, pp. 9-10, 1985.

[3] L. A. Zadeh, "Toward a theory of fuzzy information granulation and its centrality in human reasoning and fuzzy logic," Fuzzy Sets and Systems, vol. 90, no. 2, pp. 111-127, 1997.

[4] M. Atef, A. M. Khalil, S. G. Li, A. Azzam, and A. A. El Atik, "Comparison of six types of rough approximations based on j-neighborhood space and j-adhesion neighborhood space," Journal of Intelligent \& Fuzzy Systems, vol. 39, no. 3, pp. 4515-4531, 2020.

[5] M. Atef, S. Nada, A. Gumaei, and A. S. Nawar, "On three types of soft rough covering-based fuzzy sets," Journal of Mathematics, vol. 2021, Article ID 6677298, 9 pages, 2021.

[6] A. A. El Atik, A. S. Nawar, and M. Atef, "Rough approximation models via graphs based on neighborhood systems," Granular Computing, 2020.

[7] T. Herawan, M. Deris, and J. Abawajy, "A rough set approach for selecting clustering attribute," Knowledge-Based Systems, vol. 23, no. 3, pp. 220-231, 2010.

[8] G. Liu and W. Zhu, "The algebraic structures of generalized rough set theory," Information Sciences, vol. 178, no. 21, pp. 4105-4113, 2008.

[9] Y. Y. Yao, "Three-way decisions with probabilistic rough sets," Information Sciences, vol. 180, no. 3, pp. 341-353, 2010.

[10] H. Zhang, H. Liang, and D. Liu, "Two new operators in rough set theory with applications to fuzzy sets," Information Sciences, vol. 166, no. 1-4, pp. 147-165, 2004.

[11] B. Sun, W. Ma, and Y. Qian, "Multigranulation fuzzy rough set over two universes and its application to decision making," Knowledge-Based Systems, vol. 123, pp. 61-74, 2017.

[12] D. S. Yeung, Degang Chen, E. C. C. Tsang, J. W. T. Lee, and Wang Xizhao, "On the generalization of fuzzy rough sets," IEEE Transaction Fuzzy System, vol. 13, no. 3, pp. 343-361, 2005.

[13] W. Ziarko, "Variable precision rough set model," Journal of Computer and System Sciences, vol. 46, no. 1, pp. 39-59, 1993.
[14] Y. Y. Yao and B. Yao, "Covering based rough set approximations," Information Sciences, vol. 200, pp. 91-107, 2012.

[15] Z. Bonikowski, E. Bryniarski, and U. Wybraniec-Skardowska, "Extensions and intentions in the rough set theory," Information Sciences, vol. 107, no. 1-4, pp. 149-167, 1998.

[16] W. Zhu and F. Wang, "On three types of covering-based rough sets," IEEE Transaction Knowledge Data Engineering, vol. 19, no. 8, pp. 1131-1144, 2007.

[17] M. Atef and A. A. El Atik, "Some extensions of covering-based multigranulation fuzzy rough sets from new perspectives," Soft Computing, vol. 25, no. 8, pp. 6633-6651, 2021.

[18] M. Atef and A. A. Azzam, "Covering fuzzy rough sets via variable precision," Journal of Mathematics, vol. 2021, Article ID 5525766, 10 pages, 2021.

[19] J. Ma, M. Atef, S. Nada, and A. Nawar, "Certain types of covering-based multigranulation $\mathscr{I}, \mathscr{T}$-fuzzy rough sets with application to decision-making," Complexity, vol. 2020, Article ID 6661782, 20 pages, 2020.

[20] Y. H. Qian and J. Y. Liang, "Rough set method based on multigranulations," in 2006 5th IEEE International Conference on Cognitive Informatics, pp. 297-304, Beijing, China, 2006.

[21] Y. H. Qian, J. Y. Liang, Y. Y. Yao, and C. Y. Dang, "MGRS: a multi-granulation rough set," Information Sciences, vol. 180, no. 6, pp. 949-970, 2010.

[22] Y. H. Qian, J. Y. Liang, and C. Y. Dang, "Incomplete multigranulation rough set," IEEE Transactions on Systems, Man, and Cybernetics - Part A: Systems and Humans, vol. 40, no. 2, pp. 420-431, 2010.

[23] Y. H. Qian, J. Y. Liang, W. Pedrycz, and C. Y. Dang, "Positive approximation: an accelerator for attribute reduction in rough set theory," Artificial Intelligence, vol. 174, no. 9-10, pp. 597$618,2010$.

[24] X. B. Yang, X. N. Song, Z. H. Chen, and J. Y. Yang, "On multigranulation rough sets in incomplete information system," International Journal of Machine Learning and Cybernetics, vol. 3, no. 3, pp. 223-232, 2012.

[25] X. B. Yang, X. N. Song, H. L. Dou, and J. Y. Yang, "Multi-granulation rough set:from crisp to fuzzy case," Ann. Fuzzy Math. Inform., vol. 1, no. 1, pp. 55-70, 2011.

[26] W. H. Xu, Q. R. Wang, and X. T. Zhang, "Multi-granulation fuzzy rough set model on tolerance relations," in Proceedings of the Fourth International Work shop on Advanced Computational Intelligence, pp. 357-364, Wuhan, Hubei, China, 2006.

[27] W. H. Xu, Q. R. Wang, and S. Luo, "Multi-granulation fuzzy rough sets," Journal of Intelligent \& Fuzzy Systems, vol. 26, no. 3, pp. 1323-1340, 2014.

[28] W. H. Xu, Q. R. Wang, and X. T. Zhang, "Multi-granulation fuzzy rough sets in a fuzzy tolerance approximation space," International Journal of Fuzzy System, vol. 13, no. 4, pp. 246259, 2011.

[29] H. She and X. L. He, "On the structure of the multigranulation rough set model," Knowledge-Based Systems, vol. 36, pp. 8192, 2012.

[30] J. Y. Liang, F. Wang, C. Y. Dang, and Y. H. Qian, “An efficient rough feature selection algorithm with a multi-granulation view," International Journal of Approximate Reasoning, vol. 53, no. 6, pp. 912-926, 2012.

[31] C. H. Liu and D. Q. Miao, "Covering rough set model based on multi-granulations," in Proceedings of the Thirteenth International Conference on Rough Sets, Fuzzy Set, Data Mining and 
Granular Computing, in:LNCS(LNAI), vol. 6743, pp. 87-90, Moscow, Russia, 2011.

[32] C. H. Liu and M. Z. Wang, "Covering fuzzy rough set based on multi-granulations," in Proceedings of International Conference on Uncertainty Reasoning and Knowledge Engineering, vol. 2, pp. 146-149, Bali, Indonesia, 2011.

[33] G. P. Lin, Y. H. Qian, and J. J. Li, "NMGRS: neighborhoodbased multigranulation rough sets," International Journal of Approximate Reasoning, vol. 53, no. 7, pp. 1080-1093, 2012.

[34] G. P. Lin, J. Y. Liang, and Y. H. Qian, "Multigranulation rough sets: from partition to covering," Information Sciences, vol. 241, pp. 101-118, 2013.

[35] W. H. Xu, W. X. Sun, X. Y. Zhang, and W. X. Zhang, "Multiple granulation rough set approach to ordered information systems," International Journal of General Systems, vol. 41, no. 5, pp. 475-501, 2012.

[36] W. H. Xu, Q. R. Wang, and X. T. Zhang, "Multi-granulation rough sets based on tolerance relations," Soft Computing, vol. 17, no. 7, pp. 1241-1252, 2013.

[37] W. H. Xu, X. T. Zhang, and Q. R. Wang, "A generalized multigranulation rough set approach," in Bio-Inspired Computing and Applications, vol. 6840, pp. 681-689, Springer Berlin Heidelberg, Berlin, Heidelberg, 2012.

[38] Y. H. Qian, H. Zhang, Y. L. Sang, and J. L. Liang, "Multigranulation decision-theoretic rough sets," International Journal of Approximate Reasoning, vol. 55, no. 1, pp. 225-237, 2014.

[39] C. Liu, D. Miao, and J. Qian, "On multi-granulation covering rough sets," International Journal of Approximate Reasoning, vol. 55, no. 6, pp. 1404-1418, 2014.

[40] Q. Kong and $\mathrm{W} . \mathrm{Xu}$, "The comparative study of covering rough sets and multi-granulation rough sets," Soft Computing, vol. 23, no. 10, pp. 3237-3251, 2019.

[41] C. Liu and W. Pedrycz, "Covering-based multi-granulation fuzzy rough sets," Journal of intelligent \& fuzzy systems, vol. 30, no. 1, pp. 303-318, 2016.

[42] P. Liu, A. Ali, and N. Rehman, "Multi-granulation fuzzy rough sets based on fuzzy preference relations and their applications," IEEE Access, vol. 7, pp. 147825-147848, 2019.

[43] L. Sun, L. Wang, W. Ding, Y. Qian, and J. Xu, "Neighborhood multi-granulation rough sets-based attribute reduction using Lebesgue and entropy measures in incomplete neighborhood decision systems," Knowledge-Based Systems, vol. 192, article 105373, 2020.

[44] M. Liang, J. Mi, T. Feng, and B. Xie, "A dynamic approach for updating the lower approximation in adjustable multigranulation rough sets," Soft Computing, vol. 24, no. 21, pp. 15951-15966, 2020.

[45] M. E. Abd El-Monsef, A. M. Kozae, and M. K. El-Bably, "On generalizing covering approximation space," Journal of Egyptian Mathematical Society, vol. 23, no. 3, pp. 535-545, 2015.

[46] B. De Baets and E. Kerre, "A revision of Bandler-Kohout compositions of relations," Mathematica Pannonica, vol. 4, no. 1, pp. 59-78, 1993.

[47] B. Yang and B. Q. Hu, "Fuzzy neighborhood operators and derived fuzzy coverings," Fuzzy Sets and Systems, vol. 370, pp. 1-33, 2019.

[48] X. You, J. Li, and H. Wang, "Relative reduction of neighborhood-covering pessimistic multigranulation rough set based on evidence theory," Information, vol. 10, no. 11, p. $334,2019$.
[49] J. Ma, M. Atef, A. M. Khalil, N. Hassan, and G. -X. Chen, "Novel models of fuzzy rough coverings based on fuzzy $\alpha$ -neighborhood and its application to decision-making," IEEE Access, vol. 8, pp. 224354-224364, 2020.

[50] A. S. Nawar, M. Atef, and A. M. Khalil, "Certain types of fuzzy soft $\beta$-covering based fuzzy rough sets with application to decision-making," Journal of Intelligent \& Fuzzy Systems, vol. 40, no. 6, pp. 10825-10836, 2021.

[51] M. Atef and S. I. Nada, "On three types of soft fuzzy coverings based rough sets," Mathematics and Computers in Simulation, vol. 185, pp. 452-467, 2021. 